\title{
ANALYTICAL TECHNIQUES FOR AMBIENT SULFATE AEROSOLS
}

ANL-81-12

by

S. A. Johnson, D. G. Graczyk, R. Kumar, and $P$. T. Cunningham

ARGONNE NATIONAL LABORATORY, ARGONNE, ILLINOIS

Prepared for the U. S. DEPARTMENT OF ENERGY

under Contract W-31-109-Eng-38 
The facilities of Argonne National Laboratory are owned by the United States Government. Under the terms of a contract (W-31-109-Eng-38) among the U. S. Department of Energy, Argonne Universities Association and The University of Chicago, the University employs the staff and operates the Laboratory in accordance with policies and programs formulated, approved and reviewed by the Association.

\section{MEMBERS OF ARGONNE UNIVERSITIES ASSOCIATION}

The University of Arizona Carnegie-Mellon University Case Western Reserve University The University of Chicago University of Cincinnati Illinois Institute of Technology University of Illinois Indiana University The University of Iowa Iowa State University
The University of Kansas Kansas State University Loyola University of Chicago Marquette University The University of Michigan Michigan State University University of Minnesota University of Missouri Northwestern University University of Notre Dame
The Ohio State University

Ohio University

The Pennsylvania State University

Purdue University

Saint Louis University

Southern Illinois University

The University of Texas at Austin

Washington University

Wayne State University

The University of Wisconsin-Madison

This report was prepared as an account of work sponsored by an agency of the United States Government. Neither the United States Government or any agency thereof, nor any of their employees, make any warranty, express or implied, or assume any legal liability or responsibility for the accuracy, completeness, or usefulness of any information, apparatus, product, or process disclosed, or represent that its use would not infringe privately owned rights. Reference herein to any specific commercial product, process, or service by trade name, mark, manufacturer, or otherwise, does not necessarily constitute or imply its endorsement, recommendation, or favoring by the United States Government or any agency thereof. The views and opinions of authors expressed herein do not necessarily state or reflect those of the United States Government or any agency thereof.

Printed in the United States of America

Available from

National Technical Information Service

U. S. Department of Commerce

5285 Port Royal Road

Springfield, VA 22161

NTIS price codes

Printed copy: $\mathrm{A} 03$

Microfiche copy: A01 


\section{DISCLAIMER}

This report was prepared as an account of work sponsored by an agency of the United States Government. Neither the United States Government nor any agency Thereof, nor any of their employees, makes any warranty, express or implied, or assumes any legal liability or responsibility for the accuracy, completeness, or usefulness of any information, apparatus, product, or process disclosed, or represents that its use would not infringe privately owned rights. Reference herein to any specific commercial product, process, or service by trade name, trademark, manufacturer, or otherwise does not necessarily constitute or imply its endorsement, recommendation, or favoring by the United States Government or any agency thereof. The views and opinions of authors expressed herein do not necessarily state or reflect those of the United States Government or any agency thereof. 


\section{DISCLAIMER}

Portions of this document may be illegible in electronic image products. Images are produced from the best available original document. 
Distribution Category:

Environmental Control Technology

and Earth Sciences (UC-11)

ANL-81-12

\begin{abstract}
ARGONNE NATIONAI LABORATORY
9700 South Cass Avenue

Argonne, I11inois 60439
\end{abstract}

\title{
ANALYTICAL TECHNIQUES FOR AMBIENT SULFATE AEROSOLS
}

by

S. A. Johnson, D. G. Graczyk, R. Kumar, and $P$. T. Cunningham

Chemical Engineering Division

June 1981

This book was prepared as an account of work sponsored by an dgency of the Unized States Government Netther the United Sistes Government nar any axpeney thereof rior any of their employers rrakes ary warranty express of impled or assumce anty legal leablilty or responsibitity for the accuracy completeness or usefuness of any intormation apparetus protuct or process diseksed of commercial product urocess or service by trate name rademark manufact herer ot othy spectic

not necessarily construte or imoly its endotsement recommendation or favoring by the united

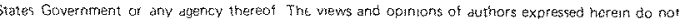
necessarily statte or reflect thase of the Ufrtited States Government or any agency thereot 


.


ABSTRACT . . . . . . . . . . . . . . . . . . . . 1

I. INTRODUCTION ..................... . . . 2

II. FILTER-EXTRACTION METHOD FOR SULFATE ANALYSIS . . . . . . . 2

A. Filter Handing and Extraction . . . . . . . . . . 3

B. Sulfate Standardization ................. 3

III. RESULTS OF FIELD STUDIES . . . . . . . . . . . . . . . 5

A. Comparison of Sampling Techniques . . . . . . . . . 5

B. Measurement of Sulfate Acidity at Tyson, MO . . . . . . 12

IV. DEVELOPMENT OF A NEW INSTRUMENT AND PROCEDURE . . . . . . . . 15

A. The ATR-Impactor Device . . . . . . . . . . 15

1. Background . . . . . . . . . . 15

2. Design . . . . . . . . . . . . . . . 15

3. Results and Discussion .......... 18

a. Initial Field Trials . . . . . . . . . . 18

b. Airborne-Plume Studies ............ 22

c. General Observations ............ 23

4. Summary ................. . . 23

B. X-Ray Fluorescence Method for the Determination

of Proton Acidity in Aerosol Sulfates......... 23

1. Reagent Selection ............. 24

2. Discussion of Experimental Procedures ....... 25

a. Impactor Samples ........... . . 25

b, Filter Samples ............. 29

3. Summary .............. . . 32

ACKNOWLEDGMENTS . . . . . . . . . . . . . . . . . . . 33

REFERENCES . . . . . . . . . . . . . . . . . . . 34 


\section{LIST OF FIGURES}

No.

1. Beer's Law Plot for Ammonium Sulfate Standards Prepared by Freeze-Drying . . . . . . . . . . . . . . . . . .

2. Comparison of Results from Infrared Analysis of EPA Fine Filter Samples and ANL Stage-IV Lundgren Impactor Samples, Plotted as $\% \mathrm{SO}_{4}^{2-}$ of the Total Sample Weight . . . . . . . .

3. Comparison of Results from Infrared Analysis of EPA Fine Filter Samples and ANL Stage-IV Lundgren Impactor Samples, Plotted as $\mu \mathrm{g} \mathrm{SO} \mathrm{S}_{4}^{2-} / \mathrm{m}^{3} \mathrm{Air}$. . . . . . . . . . . . . 10

4. Schematic Diagram of the ATR-Impactor . . . . . . . . 17

5. Detail of Nozzles and ATR Plates............ 18

6. Infrared Spectra of Ambient Aerosol Samples Collected Simultaneously to Illustrate the Greater Sensitivity of the ATR Method vs, the $\mathrm{KBr}$-Pellet Method ...........

7. Infrared Spectra of an Ambient Aerosol Sample Immediately after Collection and after Six-Months Storage . . . . . . . 20

8. Infrared Spectra of Submicrometer Ambient Aeroso1 Collected with ATR-Impactor . . . . . . . . . . . .

9. Infrared Spectra of Power-Plant Plume Samples Collected with ATR-Impactor . . . . . . . . . . . . . . .

10. Calibration of Filter Samples for Sulfur Determination by the XRF Method . . . . . . . . . . . . . . . .

11. Results of the Neutralization of Sulfuric Acid by TAPG on Fluoropore Filters . . . . . . . . . . . . . . . . 


\section{LIST OF TABLES}

No.

1. Sulfate Found on EPA Filter Samples . . . . . . . . . . .

2. Results of Analysis of Stage-IV Lundgren Impactor Samples

Collected by ANL during the Comparison Study . . . . . . . . .

3. Analysis of Stage-IV Lundgren Impactor Samples Collected by ANL at Tyson, MO . . . . . . . . . . . . . . . . . .

4. Sumnary of Data from Analysis of Field Samples of Sulfate Aerosol . . . . . . . . . . . . . . .

5. Results of Analysis of Synthetic Sulfate Aerosol on Filters Neutralized with TAPG in a Closed Chamber . . . . . .

6. Results of Analysis of Synthetic Sulfate Aerosol on Filters Neutralized with TAPG in Flow-Through Procedure . . . 


\section{ANALYTICAL TECHNIQUES FOR AMBIENT \\ SULFATE AEROSOLS}

by

S. A. Johnson, D. G. Graczyk, R. Kumar, and $P$. T. Cunningham

\section{ABSTRACT}

This report describes work done to further develop the infrared spectroscopic analytical method for the analysis of atmospheric aerosol particles, as wel1 as some exploratory work on a new procedure for determining proton acidity in aerosol samples.

Earliex work had led to the successful use of infrared (IR) spectrophotometry for the analysis of nitrate, ammonium, and neutral and acidic sulfates in aerosol samples collected by an impactor on a Mylar-film substrate. In this work, a filterextraction method was developed to prepare filter-collected aerosol samples for IR analysis. A study was made comparing the IR analytical results on filter-collected samples with impactorcollected samples. Also, the infrared analytical technique was compared in field studies with light-scattering techniques for aerosol analysis.

A highly sensitive instrument for aerosol analysis using attenuated total internal reflection (ATR) infrared spectroscopy was designed, built, and tested. This instrument provides a measurement sensitivity much greater (by a factor of 6 for $\mathrm{SO}_{4}^{2-}$ ) than that obtainable using the $\mathrm{KBr}$-pellet method. This instrument collects size- and time-resolved samples and is potentially capable of providing automated, near real-time aerosol analysis.

Finally, this report describes some exploratory work on a novel approach to the determination of proton acidity in filteror impactor-collected aerosol samples. In this technique, the acidic sample is reacted with an excess of a tagged, vapor-phase base. The unreacted base is flushed off and the amount of the tag retained by the sample is a direct measure of the proton acidity of the sample. In this work, the base was tagged with $\mathrm{Ge}$, which can be conveniently determined by the X-ray fluorescence technique. 


\section{INTRODUCTION}

We have developed a method for the chemical characterization and analysis of airborne particulate material as a function of particle size and time. Our technique, which is based on the infrared (IR) spectroscopic analysis of particulate samples, has been used to show that there are, indeed, significant variations in the chemistry of airborne particulate with size and that, within a specific size range, the chemistry does change with time.1,2 of particular significance has been the identification of ammonium sulfate as the predominant constituent of submicrometer particles and the observation that the degree of acidity of this sulfate is highly variable with time. This work has been directed toward further development of the infrared spectroscopic method, as we11 as other techniques for the detailed characterization and analysis of atmospheric neutral and acidic sulfates.

The infrared characterization of airborne particulate material has been developed to the point where quantitative determination of neutral sulfate is possible for samples containing from one to several hundred micrograms of this ion. Systematic variations of absorption-band position and intensity in the spectra of some sulfate samples are associated with the degree of acidity of the sample. This infrared method permits analysis of sulfate in samples that have been collected on Mylar film using an inertial impactor.

This report describes the work conducted in three distinct, but interrelated, program areas. The first was a laboratory effort to develop a procedure whereby samples collected on filters may also be analyzed using the previously developed infrared spectrophotometric method. The second element was participation in two field studies for the comparison of various sampling and analytical methods for the determination of ambient sulfates. Finally, this report describes development work on a new instrument and a new procedure for the measurement of atmospheric sulfates with increased sensitivity.

\section{FILTER-EXTRACTION METHOD FOR SULFATE ANALYSIS}

Airborne particulate matter is commonly collected by a variety of filtration and impaction methods, each having its advantages and disadvantages. At ANL, samples for infrared analysis are collected with a Lundgren impactor.1,2 The design of the impactor facilitates sample handiing and supplies the necessary time- and size-resolution. The particles are classified into four size ranges during collection, while a controlled rate of movement of the collecting substrate past the impaction nozzles provides the time-resolution needed. The use of the impaction principle, however, limits the sample to those particles larger than $\sim 0.3-\mu \mathrm{m}$-dia. To extend the capability of the infrared spectroscopic method to particles smaller than $0.3-\mu \mathrm{m}$-dia, a method was needed to recover the sulfate from the impactor after-filter.

Previous procedures for analyzing sulfate via filter-extraction recovery resulted in neutralization of the acid sulfate. A new procedure, using concepts like those of Citron and Underwood, ${ }^{3}$ has been investigated wherein sulfate can be quantitatively recovered from filter-collected samples while apparently maintaining the acidity of the sample. The new procedure and the sulfate standardization are described below. 


\section{A. Filter Handling and Extraction}

The procedure starts with the extraction of the water-soluble material from the filter by ultrasonic agitation of the filter in $10-20 \mathrm{~mL}$ of distilled, $\mathrm{CO}_{2}$-free water. After several minutes of ultrasonic agitation in a freeze-drying flask, the filter is removed and saved for a second extraction if desired. In preliminary tests the second extract of the filters was found to be free of sulfate and one extraction was determined to be sufficient. A weighed amount of potassium bromide is added to the extract in the flask, and the solution is immediately frozen by partially immersing the flask in liquid nitrogen. The water is removed by freeze-drying, which takes 4-8 h. The dry sample is removed from the flask and pressed into a pellet for subsequent infrared spectroscopic examination. Samples of ammonium acid sulfate aerosol, produced using a flow reactor to simulate ambient conditions, were collected on Fluoropore filters (Teflon, Type FALP-3700, Millipore Corporation) and extracted using this freeze-drying procedure with good results. In preliminary tests, the acidity of the sulfate was maintained throughout the procedure without any apparent neutralization or reaction with the $\mathrm{KBr}$. Some material is lost during transfer from the freeze-drying flask; therefore, the $\mathrm{KBr}$ is weighed again to account for these losses. Care must be taken in handling and storage of the filters to prevent neutralization prior to the extraction. The possibility exists that neutralization could take place during the collection process. This has not been a problem with impacted samples but may be with filter samples since the collected particles have greater exposure to the gas stream. An ammonia denuder in the incoming gas stream may be one method to alleviate this, should it prove to be a problem.

However, subsequent work using reagent-grade ammonium bisulfate to prepare the standards showed that the freeze-drying process for the filter extract may not always maintain the acidity of the original sample. Depending upon the freeze-drying conditions ( $. g .$, temperature of the sample and time for drying), the loss of $\mathrm{NH}_{3}$ and $\mathrm{H}^{+}$, along with water from the freeze-drying matrix, may alter the acidity of the dried sample. This problem has not been completely resolved for samples of acidic ammonium sulfates, but this aqueous extraction/freeze-drying procedure does give acceptable results for neutral ammonium sulfate samples.

\section{B. Sulfate Standardization}

Quantification for sulfate was accomplished by preparing standard solutions containing $43.10 \mathrm{mg} / \mathrm{L}$ and $65.44 \mathrm{mg} / \mathrm{L}$ of "ultra-pure" $99.99 \%$ ammonium sulfate (Research Organic/Inorganic Chemical Corp., Sun Valley, CA). Aliquots were pipetted into the freeze-drying flasks, and the procedure described above for filter samples was followed for the standards. The results for these standards are presented in Fig. I, which is a Beer's law plot of the absorbance for the $1400-\mathrm{cm}^{-1} \mathrm{NH}_{4}^{+}$band and the $1110-\mathrm{cm}^{-1}$ and $620-\mathrm{cm}^{-1} \mathrm{SO}_{4}^{2-}$ bands. The lines represent a least-squares fit of the data. As can be seen, straight-line relationships are achieved over the range of sample weights. The correlation coefficients and the values of the absolute absorbance for the three bands are: 


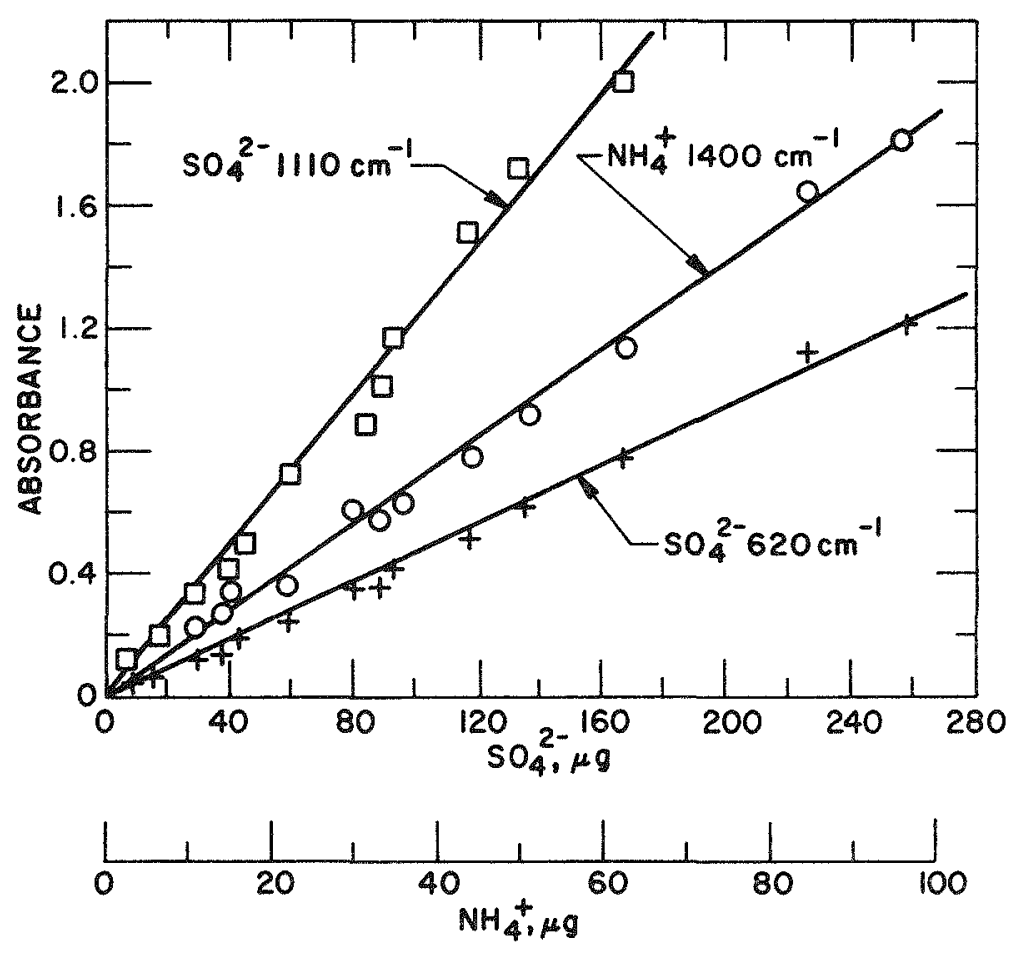

Fig. 1. Beer's Law P1ot for Ammonium Sulfate Standards Prepared by Freeze-Drying

$\mathrm{SO}_{4}^{2-}, 620-\mathrm{cm}^{-1}$ band

Absolute Absorbance $=4.67 \times 10^{-3} \times \mu \mathrm{g} \mathrm{SO} \mathrm{S}^{2-}$

Correlation Coefficient $=0.999$

$\mathrm{SO}_{4}^{2-}, 1110-\mathrm{cm}^{-1}$ band

Absolute Absorbance $=12.17 \times 10^{-3} \times \mu \mathrm{g} \mathrm{SO}_{4}^{2-}$

Correlation Coefficient $=0.997$

$\mathrm{NH}_{4}^{+}, 1400-\mathrm{cm}^{-1}$ band

Absolute Absorbance $=1.86 \times 10^{-2} \times \mu \mathrm{g} \mathrm{NH} \mathrm{NH}^{+}$

Correlation Coefficient $=0.999$.

The $620-\mathrm{cm}^{-1}$ band is recommended for analytical use since it is relatively free from interference in ambient samples and since it does not have slopingbaseline problems commonly found in spectra from cloudy $\mathrm{KBr}$ pellets. 


\section{RESULTS OF FIELD STUDIES}

In order to compare the results from our procedure of impactor collection and infrared spectroscopic analysis with the results obtained by other sampling and analytical techniques, we participated in two separate field studies where samples were collected simultaneously by several different techniques for in situ or subsequent analysis by the respective investigators.

\section{A. Comparison of Sampling Techniques}

During August and September of 1975, a field comparison study was conducted in the St. Louis region in coordination with the Environmental Protection Agency (EPA). The sampling phase of the study entailed the simultaneous collection of airborne particulate matter by ANL, the EPA, and other participants, at both a rural and an urban site. Only the samples obtained by ANL and the EPA are discussed here. The ANL samples were collected using Lundgren impactors with a time resolution of $2 \mathrm{~h}$, while the EPA samples* were collected on 37-mm-dia Fluoropore (Teflon) filters using a dichotomous sampler with a time resolution of $24 \mathrm{~h}$. The results on the samples from Stage IV of the Lundgren impactor (size range 0.3 to $1.0-\mu m$ aerodynamic diameter) and the coarse $(>3.5-\mu \mathrm{m}$-dia) and the fine $(<3.5-\mu m$-dia) size fractions from the dichotomous sampler have been compared.

Table 1 presents the sulfate-analysis results for the EPA filters prepared by the freeze-drying procedure and then analyzed by infrared spectroscopy as described in II-A and II-B above. The sample weights were determined gravimetrically by the EPA. The values for loading of the air are calculated from these weights and the sampling air flow rates. The sulfate values are presented in three ways: total weight of sulfate per filter ( $\mu g)$, percent of the mass collected that is sulfate $(\%)$, and loading of the sulfate in the air $\left(\mu \mathrm{g} / \mathrm{m}^{3}\right)$. In all but three of the coarse-particle samples, either the sulfate values were close to our limit of detection by IR, or the infrared spectra had interfering bands; therefore, no values for sulfate are listed for most of the coarse-particle samples. Table 2 gives the sampling and analytical data on the samples collected by ANL on Stage-IV of the Lundgren impactor in 2-h collection periods. (Stage IV collects particles from $0.3 \mu \mathrm{m}$ to $1.0 \mu \mathrm{m}$ aerodynamic diameter.)

Figures 2 and 3 are plots of the results for sulfate from the Stage-IV impactor samples and the dichotomous fine filter samples for the period Aug. 28 to Sept. 4, 1975. In Fig. 2, the amount of sulfate is plotted as weight percent of the total sample. The points indicate the values for the 2-h impactor samples and the bars indicate the values for the $24-h$ EPA filter samples. Figure 3 presents the same data plotted as $\mu \mathrm{g} \mathrm{SO}_{4}^{2-} / \mathrm{m}^{3}$ air. As can be seen in the \% $\mathrm{SO}_{4}^{2-}$ plot, Fig. 2, the values for the filter samples are somewhat higher but generally in the same range as those for the impactor samples; when sulfate is plotted as $\mu \mathrm{g} / \mathrm{m}^{3}, \mathrm{Fig} .3$, the filter values are substantially higher than the impactor values. These trends are consistent with the current understanding of the size distribution and chemical

\footnotetext{
The thirty EPA samples were supplied to us by T. Dzubay, EPA.
} 
Table 1. Sulfate Found on EPA Filter Samples

\begin{tabular}{|c|c|c|c|c|c|c|c|}
\hline \multirow[b]{2}{*}{$\begin{array}{c}\text { Collection } \\
\text { Date }\end{array}$} & \multirow[b]{2}{*}{$\begin{array}{l}\text { Particle } \\
\text { Size }\end{array}$} & \multirow[b]{2}{*}{$\begin{array}{c}\text { EPA } \\
\text { Filter } \\
\text { Number }\end{array}$} & \multirow[b]{2}{*}{$\begin{array}{l}\text { Sample } \\
\text { Mass, } \\
\mu g\end{array}$} & \multirow{2}{*}{$\begin{array}{l}\text { Sample } \\
\text { Loading } \\
\text { in Air, } \\
\mu g / \mathrm{m}^{3}\end{array}$} & \multicolumn{3}{|c|}{ Sulfate Analysis } \\
\hline & & & & & $\begin{array}{c}\text { Wt/Filter, } \\
\mu \mathrm{g}\end{array}$ & $\begin{array}{l}\text { Percent } \\
\text { of Mass, } \\
\%\end{array}$ & $\begin{array}{c}\text { Sulfate Loading } \\
\text { in Air, } \\
\mu \mathrm{g} / \mathrm{m}^{3}\end{array}$ \\
\hline & & & & Urban & & & \\
\hline Sun., Aug. 24 & $\begin{array}{l}\text { Fine } \\
\text { Coarse }\end{array}$ & $\begin{array}{r}207 \\
5207\end{array}$ & $\begin{array}{l}300 \\
300\end{array}$ & $\begin{array}{l}15.5 \\
15.5\end{array}$ & $\begin{array}{c}20.6 \\
-\end{array}$ & $\begin{array}{c}6.9 \\
-\end{array}$ & $\begin{array}{c}1.1 \\
-\end{array}$ \\
\hline Mon., Aug. 25 & $\begin{array}{l}\text { Fine } \\
\text { Coarse }\end{array}$ & $\begin{array}{r}208 \\
5208\end{array}$ & $\begin{array}{l}270 \\
400\end{array}$ & $\begin{array}{l}13.7 \\
20.2\end{array}$ & $\begin{array}{c}31.7 \\
-\end{array}$ & $\begin{array}{c}11.7 \\
-\end{array}$ & $\begin{array}{c}1.6 \\
-\end{array}$ \\
\hline Tues., Aug. 26 & $\begin{array}{l}\text { Fine } \\
\text { Coarse }\end{array}$ & $\begin{array}{c}209 \\
-\end{array}$ & $\begin{array}{c}580 \\
-\end{array}$ & $\begin{array}{c}33.3 \\
-\end{array}$ & $\begin{array}{c}75.8 \\
-\end{array}$ & $\begin{array}{c}13.1 \\
-\end{array}$ & $\begin{array}{c}4.4 \\
-\end{array}$ \\
\hline Thur., Aug. 28 & $\begin{array}{l}\text { Fine } \\
\text { Coarse }\end{array}$ & $\begin{array}{r}211 \\
5211\end{array}$ & $\begin{array}{l}770 \\
450\end{array}$ & $\begin{array}{l}39.5 \\
23.1\end{array}$ & $\begin{array}{r}210.5 \\
7.5\end{array}$ & $\begin{array}{r}27.3 \\
1.7\end{array}$ & $\begin{array}{c}10.8 \\
-\end{array}$ \\
\hline Fri., Aug. 29 & $\begin{array}{l}\text { Fine } \\
\text { Coarse }\end{array}$ & ${ }_{-}^{212^{a}}$ & $\begin{array}{c}170 \\
-\end{array}$ & $\begin{array}{c}26.4 \\
-\end{array}$ & $\begin{array}{c}33.2 \\
-\end{array}$ & $\begin{array}{c}19.5 \\
-\end{array}$ & $\begin{array}{c}5.2 \\
-\end{array}$ \\
\hline Sat., Aug. 30 & $\begin{array}{l}\text { Fine } \\
\text { Coarse }\end{array}$ & $\begin{array}{r}213 \\
5213\end{array}$ & $\begin{array}{l}430 \\
200\end{array}$ & $\begin{array}{l}21.7 \\
10.1\end{array}$ & $\begin{array}{c}166.0 \\
-\end{array}$ & $\begin{array}{c}38.6 \\
-\end{array}$ & $\begin{array}{c}8.4 \\
-\end{array}$ \\
\hline Sun., Aug. 31 & $\begin{array}{l}\text { Fine } \\
\text { Coarse }\end{array}$ & $\begin{array}{r}214 \\
5214\end{array}$ & $\begin{array}{l}540 \\
310\end{array}$ & $\begin{array}{l}27.9 \\
16.0\end{array}$ & $\begin{array}{c}209.2 \\
-\end{array}$ & $\begin{array}{c}38.7 \\
-\end{array}$ & $\begin{array}{c}10.8 \\
-\end{array}$ \\
\hline Wed., Sept. 3 & $\begin{array}{l}\text { Fine } \\
\text { Coarse }\end{array}$ & $\begin{array}{r}217 \\
5217\end{array}$ & $\begin{array}{l}510 \\
740\end{array}$ & $\begin{array}{l}26.5 \\
38.5\end{array}$ & $\begin{array}{c}82.0 \\
-\end{array}$ & $\begin{array}{c}16.1 \\
-\end{array}$ & $\begin{array}{c}4.3 \\
-\end{array}$ \\
\hline
\end{tabular}


Table 1. (cont $d)$

\begin{tabular}{|c|c|c|c|c|c|c|c|}
\hline \multirow[b]{2}{*}{$\begin{array}{l}\text { Collection } \\
\text { Date }\end{array}$} & \multirow[b]{2}{*}{$\begin{array}{l}\text { Particle } \\
\text { Size }\end{array}$} & \multirow[b]{2}{*}{$\begin{array}{c}\text { EPA } \\
\text { Filter } \\
\text { Number }\end{array}$} & \multirow[b]{2}{*}{$\begin{array}{c}\text { Sample } \\
\text { Mass, } \\
\mu g\end{array}$} & \multirow{2}{*}{$\begin{array}{l}\text { Sample } \\
\text { Loading } \\
\text { in Air, } \\
\mu \mathrm{g} / \mathrm{m}^{3}\end{array}$} & \multicolumn{3}{|c|}{ Sulfate Analysis } \\
\hline & & & & & $\begin{array}{c}\text { Wt/Filter, } \\
\mu \mathrm{g}\end{array}$ & $\begin{array}{l}\text { Percent } \\
\text { of } \underset{\%}{\%}\end{array}$ & $\begin{array}{c}\text { Sulfate Loading } \\
\text { in Air, } \\
\mu \mathrm{g} / \mathrm{m}^{3}\end{array}$ \\
\hline \multicolumn{8}{|c|}{ Rura1 } \\
\hline Sun., Aug. 24 & $\begin{array}{l}\text { Fine } \\
\text { Coarse }\end{array}$ & $\begin{array}{r}807 \\
5807\end{array}$ & $\begin{array}{l}290 \\
280\end{array}$ & $\begin{array}{l}15.3 \\
14.8\end{array}$ & $\begin{array}{c}47.5 \\
-\end{array}$ & $\begin{array}{c}16.4 \\
-\end{array}$ & $\begin{array}{c}2.5 \\
-\end{array}$ \\
\hline Mon., Aug. 25 & $\begin{array}{l}\text { Fine } \\
\text { Coarse }\end{array}$ & $\begin{array}{r}808 \\
5808\end{array}$ & $\begin{array}{l}180 \\
200\end{array}$ & $\begin{array}{r}9.2 \\
10.2\end{array}$ & $\begin{array}{r}19.5 \\
6.6\end{array}$ & $\begin{array}{r}10.8 \\
3.3\end{array}$ & $\begin{array}{c}1.0 \\
-\end{array}$ \\
\hline Tues., Aug. 26 & $\begin{array}{l}\text { Fine } \\
\text { Coarse }\end{array}$ & $\begin{array}{c}809 \\
-\end{array}$ & $\begin{array}{l}350 \\
-\end{array}$ & $\begin{array}{c}17.6 \\
-\end{array}$ & $\begin{array}{c}92.9 \\
-\end{array}$ & $\begin{array}{c}26.5 \\
-\end{array}$ & $\begin{array}{c}4.7 \\
-\end{array}$ \\
\hline Sat., Aug. 30 & $\begin{array}{l}\text { Fine } \\
\text { Coarse }\end{array}$ & $\begin{array}{r}813 \\
5813\end{array}$ & $\begin{array}{l}400 \\
250\end{array}$ & $\begin{array}{l}20.4 \\
12.8\end{array}$ & $\begin{array}{r}138.1 \\
5.2\end{array}$ & $\begin{array}{r}34.5 \\
2.1\end{array}$ & $\begin{array}{c}7.0 \\
-\end{array}$ \\
\hline Sun., Aug. 31 & $\begin{array}{l}\text { Fine } \\
\text { Coarse }\end{array}$ & $\begin{array}{r}814 \\
5814\end{array}$ & $\begin{array}{l}510 \\
280\end{array}$ & $\begin{array}{l}27.4 \\
15.1\end{array}$ & $\begin{array}{c}159.5 \\
-\end{array}$ & $\begin{array}{c}31.3 \\
-\end{array}$ & $\begin{array}{c}8.6 \\
-\end{array}$ \\
\hline Wed., Sept. 3 & $\begin{array}{l}\text { Fine } \\
\text { Coarse }\end{array}$ & $\begin{array}{r}817 \\
5817\end{array}$ & $\begin{array}{l}640 \\
550\end{array}$ & $\begin{array}{l}31.5 \\
27.1\end{array}$ & $\begin{array}{c}142.8 \\
-\end{array}$ & $\begin{array}{c}22.3 \\
-\end{array}$ & $\begin{array}{c}7.0 \\
-\end{array}$ \\
\hline Sat., Sept. 6 & $\begin{array}{l}\text { Fine } \\
\text { Coarse }\end{array}$ & $\begin{array}{r}820 \\
5820\end{array}$ & $\begin{array}{c}430 \\
-\end{array}$ & $\begin{array}{c}22.1 \\
-\end{array}$ & $\begin{array}{c}122.7 \\
-\end{array}$ & $\begin{array}{c}28.5 \\
-\end{array}$ & $\begin{array}{c}6.3 \\
-\end{array}$ \\
\hline
\end{tabular}

a-h sample. 
Table 2. Results of Analysis of Stage-IV Lundgren Impactor Samples Collected by ANL during the Comparison Study

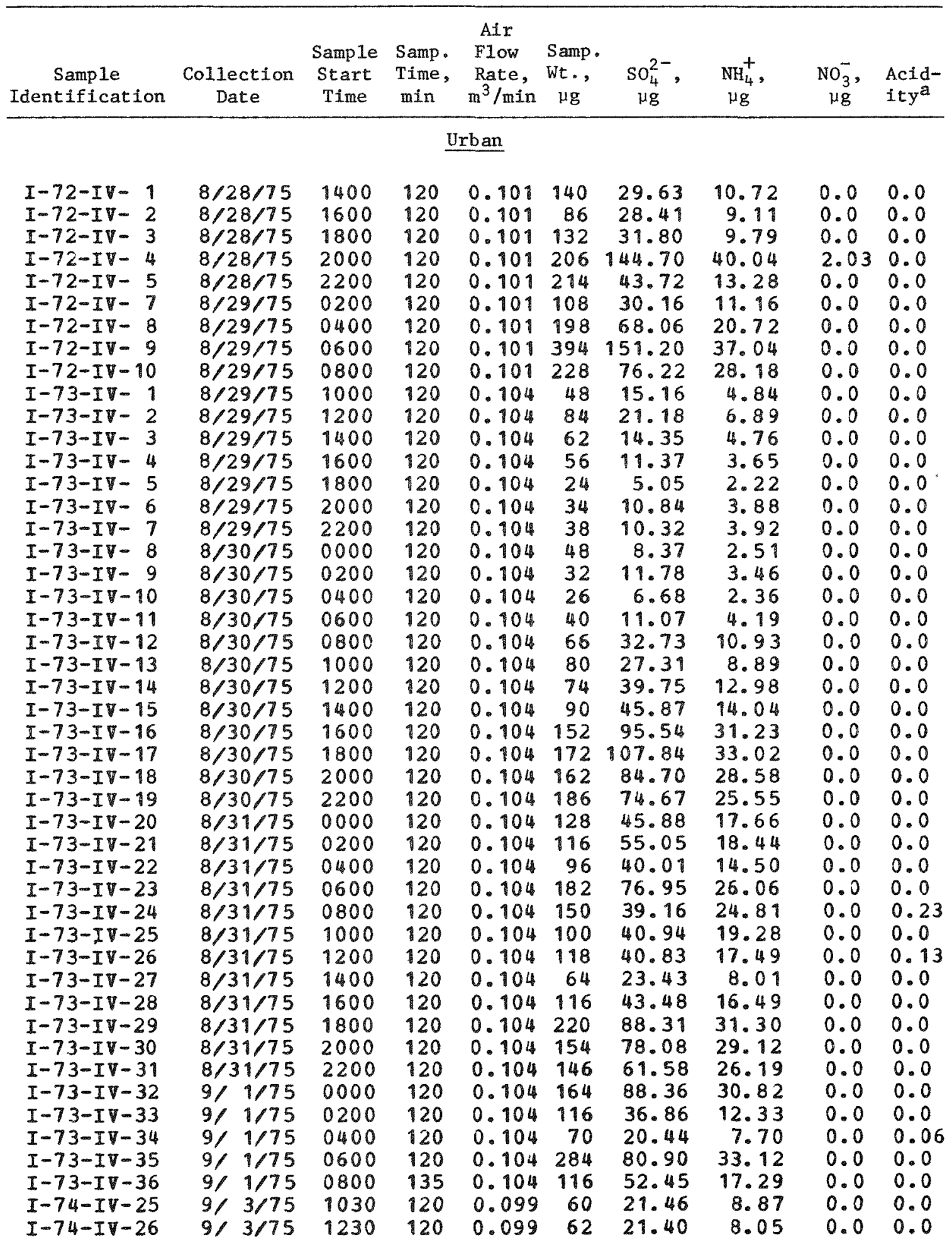

(contd) 
Table 2. (contd)

\begin{tabular}{|c|c|c|c|c|c|c|c|c|c|}
\hline $\begin{array}{c}\text { Sample } \\
\text { Identification }\end{array}$ & $\begin{array}{l}\text { Collection } \\
\text { Date }\end{array}$ & $\begin{array}{l}\text { Sample } \\
\text { Start } \\
\text { Time }\end{array}$ & $\begin{array}{l}\text { Samp, } \\
\text { Time, } \\
\text { min }\end{array}$ & $\begin{array}{l}\text { Air } \\
\text { Flow } \\
\text { Rate, } \\
\mathrm{m}^{3} / \mathrm{min}\end{array}$ & $\begin{array}{c}\text { Samp. } \\
\text { Wt. } \\
\mu g\end{array}$ & $\begin{array}{c}\mathrm{SO}_{4}^{2-} \\
\mu \mathrm{g}\end{array}$ & $\begin{array}{c}\mathrm{NH}_{4}^{+}, \\
\mu \mathrm{g}\end{array}$ & $\begin{array}{l}\mathrm{NO}_{3}^{-} \\
\mu \mathrm{g}\end{array}$ & $\begin{array}{l}\text { Acid- } \\
\text { itya }\end{array}$ \\
\hline $\begin{array}{l}I-74-I V-27 \\
I-74-I V-28 \\
I-74-I V-29 \\
I-74-I V-30 \\
I-74-I V-31 \\
I-74-I V-32 \\
I-74-I V-33 \\
I-74-I V-34 \\
I-74-I V-35 \\
I-74-I V-36\end{array}$ & $\begin{array}{ll}9 / 3 / 75 \\
9 / 3 / 75 \\
9 / 3 / 75 \\
9 / 3 / 75 \\
9 / 3 / 75 \\
9 / 4 / 75 \\
9 / 4 / 75 \\
9 / 4 / 75 \\
9 / 4 / 75 \\
9 / 4 / 75\end{array}$ & $\begin{array}{l}1430 \\
1630 \\
1830 \\
2030 \\
2230 \\
0030 \\
0230 \\
0430 \\
0630 \\
0830\end{array}$ & $\begin{array}{l}120 \\
120 \\
120 \\
120 \\
120 \\
120 \\
120 \\
120 \\
120 \\
135\end{array}$ & $\begin{array}{l}0.099 \\
0.099 \\
0.099 \\
0.099 \\
0.099 \\
0.099 \\
0.099 \\
0.099 \\
0.099 \\
0.099\end{array}$ & $\begin{array}{r}86 \\
100 \\
160 \\
106 \\
118 \\
114 \\
72 \\
94 \\
82 \\
42\end{array}$ & $\begin{array}{l}26.16 \\
30.35 \\
61.27 \\
30.26 \\
28.75 \\
14.56 \\
17.12 \\
18.82 \\
9.43 \\
9.60\end{array}$ & $\begin{array}{r}9.09 \\
11.20 \\
20.21 \\
9.23 \\
10.09 \\
4.68 \\
6.36 \\
6.16 \\
3.36 \\
3.85\end{array}$ & $\begin{array}{l}0.0 \\
0.0 \\
0.0 \\
0.0 \\
0.0 \\
0.0 \\
0.0 \\
2.58 \\
1.52 \\
0.0\end{array}$ & $\begin{array}{l}0.0 \\
0.0 \\
0.0 \\
0.0 \\
0.0 \\
0.0 \\
0.0 \\
0.0 \\
0.13 \\
0.0\end{array}$ \\
\hline
\end{tabular}

Rura1

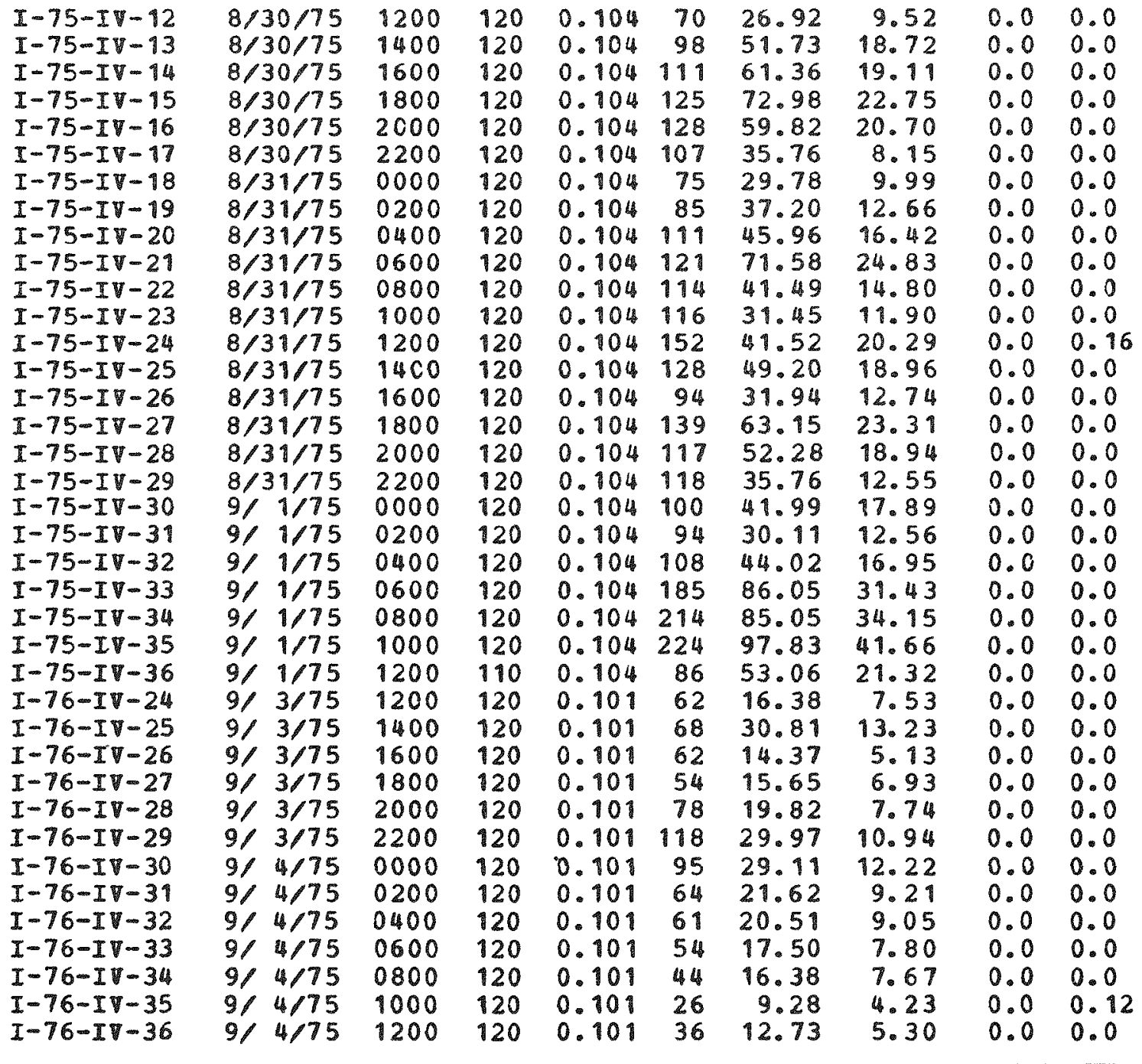

${ }^{a}$ Acidity is expressed as the molar ratio of $\mathrm{H}^{+}$to $\mathrm{SO}_{4}^{2-}$. 

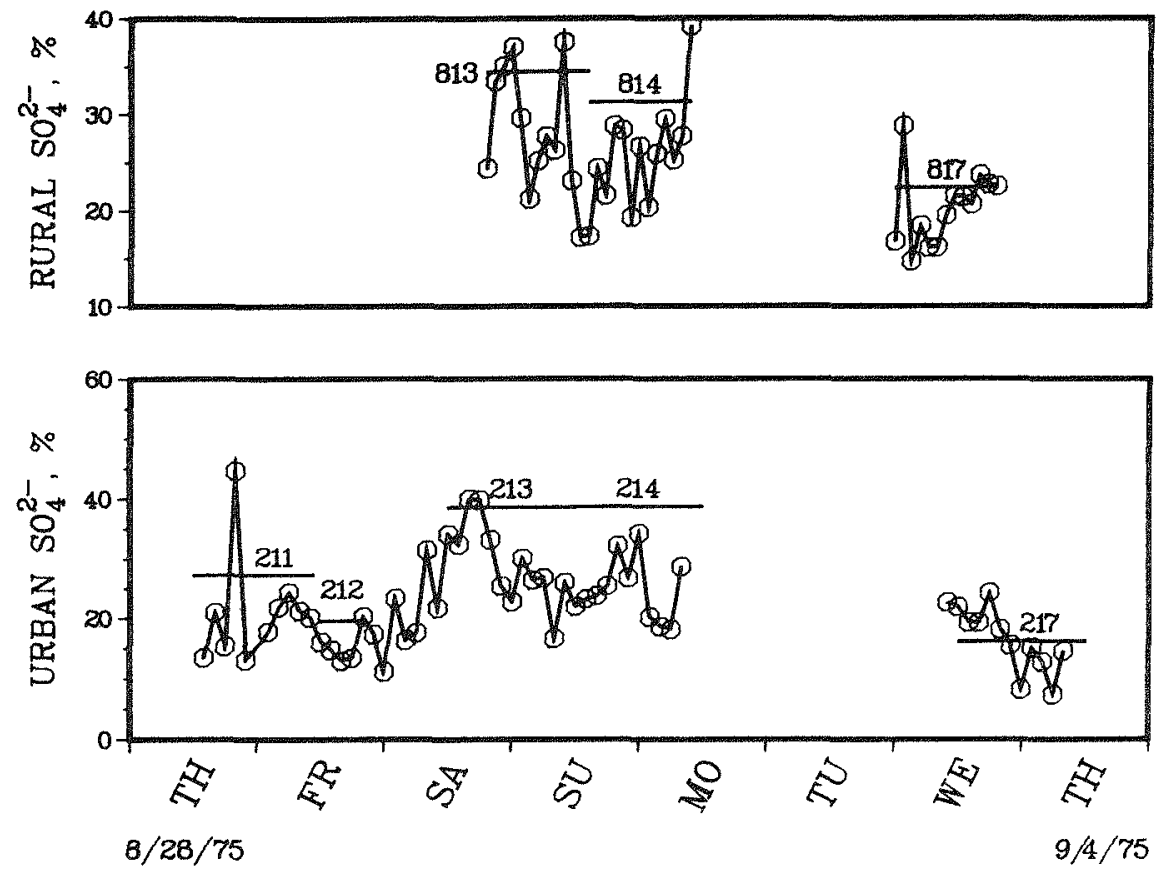

Fig. 2. Comparison of Results from Infrared Analysis of EPA Fine Filter Samples (numbered bars) and ANL Stage-IV Lundgren Impactor Samples (circles), Plotted as $\% \mathrm{SO}_{4}^{2-}$ of the Total Sample Weight.
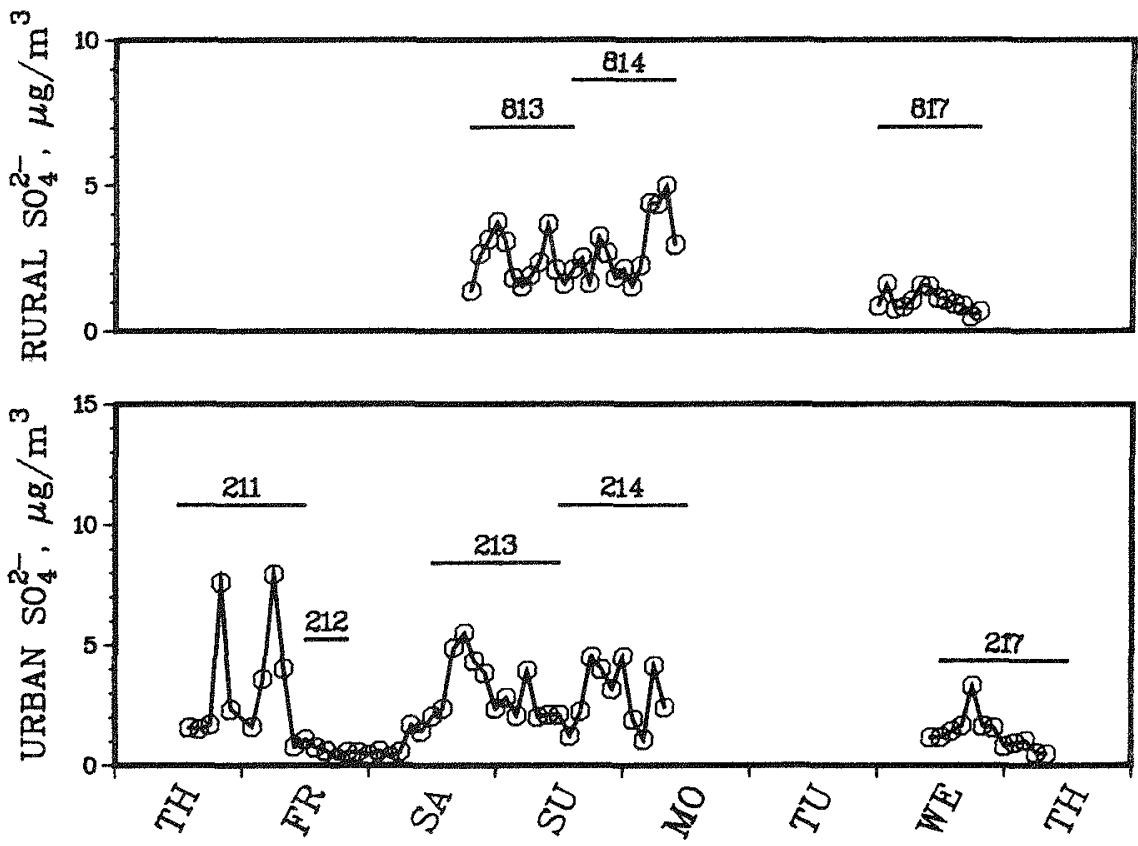

$8 / 28 / 75$

$9 / 4 / 75$

Fig. 3. Comparison of Results from Infrared Analysis of EPA Fine Filter Samples (numbered bars) and ANL Stage-IV Lundgren Impactor Samples (circles), Plotted as $\mu \mathrm{g} \mathrm{SO}_{4}^{2-} / \mathrm{m}^{3} \mathrm{Air}$. 
character of atmospheric particles; $i . e$. , they are attributed to the fact that the fine filter of the dichotomous sampler collects all particles below $3.5 \mathrm{\mu m}$ in aerodynamic diameter while stage IV of the impactor collects only a narrow size fraction between about 0.3 and $1.0 \mu \mathrm{m}$. Figure 2 then implies that the impactor-collected samples are approximately representative of the $\% \mathrm{SO}_{4}^{2-}$ to be found in the fine particles. However, since the filter collects all the material in the entire range, when plotted as $\mu \mathrm{g} \mathrm{s0} \mathrm{s}_{4}^{2-} / \mathrm{m}^{3}, \mathrm{Fig} .3$, the filter values are significantly higher than the impactor values, indicating that a substantial mass fraction, perhaps two-thirds judging from Fig. 3 , of the $\mathrm{SO}_{4}^{2-}$ passes Stage IV of the impactor and would be found on the afterfilter. It must also be pointed out that collection losses are probably greater in the impactor than in the dichotomous filter device, and this would also result in lower values for the impactor samples than for the filter samples when plotted as $\mu \mathrm{g} \mathrm{SO}_{4}^{2-} / \mathrm{m}^{3}$.

In our analysis of samples from Stage II and Stage III (nominal particle sizes collected by these two stages are 3.0 to $10.0 \mu \mathrm{m}$ and 1.0 to $3.0 \mu \mathrm{m}$, respectively) of the Lundgren impactor, we examine the upper portion of the range collected by the fine filters. The Stage-II and -III samples have very little, if any, sulfate, which indicates that the balance of sulfate found on the filters must be accounted for in the particles small enough to pass the fourth stage of the impactor and be collected on the after-filter. This demonstrates the need for the analysis of time-resolved after-filters. The development of the filter-extraction procedure now affords us this capability.

Also of note in the comparison study is the significant variation of sulfate with time as revealed by the impactor samples collected every two hours. These variations are greatly damped in the 24-h filter samples. This points out the need for time resolution as well as size resolution in atmospheric sampling if the chemistry of airborne particulate matter is to be fully understood.

The results shown in Figs. 2 and 3 also point out the need for a finer time resolution for atmospheric sampling than the 24 hours used with the dichotomous sampler. In addition to the sulfate-ion concentration fluctuating significantly during a 24-hour period, the sulfate acidity also undergoes changes that may not be detected in an aggregated 24-hour sample. For instance, Table 2 shows infrequent occurrence of acidity in impactorcollected samples, but no acidity was detected in any of the filter-collected samples. Examination of the infrared spectra of impactor samples showed significant acidity in three contiguous samples, I-73-IV-24, I-73-IV-25, and I-73-IV-26, covering a 6-hour period from 0800 to 1400 on Aug. 31, while neither of the EPA samples 213 or 214 covering the same time period showed any acidity. It should be noted that the computer analysis of the infrared spectral data did not identify sample I-73-IV-25 to be acidic (hence the acidity value of 0 in Table 2) due to an as yet unexplained shift in the absorption-band frequencies. However, visual examination of the IR absorption spectrum confirms that the sample was significantly acidic. The absence of acidity in the filter-collected samples is most likely due to their having been neutralized by basic materials collected on the filters during the balance of the 24 -hour sampling periods. Thus, a fine time resolution of 
atmospheric aerosol sampling reveals variations in the aerosol chemistry and mass concentrations that are masked when only aggregated samples are collected over 24-hour periods.

\section{B. Measurement of Sulfate Acidity at Tyson, MO}

The infrared method developed at ANL was used in a two-week field study of the occurrence of acidic sulfates at Tyson, MO. Two other research teams also participated in this study, using light-scattering techniques developed at the University of Washington, 4 Seattle and at Washington University, 5

St. Louis.

Time- and size-resolved samples were collected continuously using the Lundgren impactor for the two-week period at Tyson from September 12-26, 1975, with concurrent aerosol analysis by the light-scattering techniques. The results of the samples collected on Stage IV of the impactor are tabulated in Table 3. (As in previous field experiments, samples collected on Stages II and III had very little sulfate and little or no acidity). The data in Table 3 indicate strong acidic sulfate episodes on September 16 and 17, which were also observed by both of the 1ight-scattering techniques. The sulfate values shown in Table 3 are for the neutral sulfate fraction only and are not directly comparable to total sulfate in the case of samples with appreciable acidity.

Table 3. Analysis of Stage-IV Lundgren Impactor Samples Collected by ANL at Tyson, MO

\begin{tabular}{|c|c|c|c|c|c|c|c|c|c|}
\hline $\begin{array}{c}\text { Sample } \\
\text { Identification }\end{array}$ & $\begin{array}{l}\text { Collection } \\
\text { Date }\end{array}$ & $\begin{array}{c}\text { Sample } \\
\text { Start } \\
\text { Time }\end{array}$ & $\begin{array}{l}\text { Samp. } \\
\text { Time, } \\
\text { min }\end{array}$ & $\begin{array}{l}\text { Air } \\
\text { Flow } \\
\text { Rate, } \\
\mathrm{m}^{3} / \mathrm{min}\end{array}$ & $\begin{array}{c}\text { Samp. } \\
\text { Wt., } \\
\mu \mathrm{g}\end{array}$ & $\begin{array}{c}\mathrm{SO}_{4}^{2-}, \\
\mu \mathrm{g}\end{array}$ & $\begin{array}{c}\mathrm{NH}_{4}^{+}, \\
\mu \mathrm{g}\end{array}$ & $\begin{array}{l}\mathrm{NO}_{3}^{-} \\
\mu \mathrm{g}\end{array}$ & $\begin{array}{l}\text { Acid- } \\
\text { ity }\end{array}$ \\
\hline $\begin{array}{lr}I-77-I V- & 1+2 \\
I-77-I V- & 3+4 \\
I-77-I V- & 5+6 \\
I-77-I V- & 5+6 \\
I-77-I V- & 7+8 \\
I-77-I V-9+10 \\
I-77-I V-11+12 \\
I-77-I V-13+14 \\
I-77-I V-15+16 \\
I-77-I V-17+18 \\
I-77-I V-19+20 \\
I-77-I V-21+22 \\
I-77-I V-23+24 \\
I-77-I V-25+26 \\
I-77-I V-27+28 \\
I-77-I V-29+3 C \\
I-77-I V-31+32 \\
I-77-I V-33+34 \\
I-77-I V-35+36\end{array}$ & $\begin{array}{l}9 / 12 / 75 \\
9 / 12 / 75 \\
9 / 12 / 75 \\
9 / 12 / 75 \\
9 / 12 / 75 \\
9 / 13 / 75 \\
9 / 13 / 75 \\
9 / 13 / 75 \\
9 / 13 / 75 \\
9 / 13 / 75 \\
9 / 13 / 75 \\
9 / 14 / 75 \\
9 / 14 / 75 \\
9 / 14 / 75 \\
9 / 14 / 75 \\
9 / 14 / 75 \\
9 / 14 / 75 \\
9 / 15 / 75 \\
9 / 15 / 75\end{array}$ & $\begin{array}{l}1000 \\
1400 \\
1800 \\
1800 \\
2200 \\
0200 \\
0600 \\
1000 \\
1400 \\
1800 \\
2200 \\
0200 \\
0600 \\
1000 \\
1400 \\
1800 \\
2200 \\
0200 \\
0600\end{array}$ & $\begin{array}{l}240 \\
240 \\
240 \\
240 \\
240 \\
240 \\
240 \\
240 \\
240 \\
240 \\
240 \\
240 \\
240 \\
240 \\
240 \\
240 \\
240 \\
240 \\
236\end{array}$ & $\begin{array}{l}0.103 \\
0.103 \\
0.103 \\
0.103 \\
0.103 \\
0.103 \\
0.103 \\
0.103 \\
0.103 \\
0.103 \\
0.103 \\
0.103 \\
0.103 \\
0.103 \\
0.103 \\
0.103 \\
0.103 \\
0.103 \\
0.103\end{array}$ & $\begin{array}{r}2 \\
18 \\
90 \\
90 \\
34 \\
22 \\
18 \\
12 \\
8 \\
8 \\
48 \\
46 \\
48 \\
34 \\
14 \\
34 \\
38 \\
54 \\
78\end{array}$ & $\begin{array}{l}1.21 \\
0.0 \\
0.86 \\
0.91 \\
0.0 \\
0.0 \\
1.19 \\
0.0 \\
0.0 \\
0.0 \\
2.94 \\
0.0 \\
5.26 \\
4.57 \\
2.77 \\
8.53 \\
4.69 \\
11.64 \\
19.20\end{array}$ & $\begin{array}{l}0.96 \\
0.73 \\
0.32 \\
0.20 \\
0.0 \\
0.86 \\
1.08 \\
0.0 \\
0.0 \\
0.34 \\
2.26 \\
0.0 \\
3.19 \\
2.30 \\
1.51 \\
3.59 \\
2.58 \\
5.00 \\
8.12\end{array}$ & $\begin{array}{r}0.0 \\
0.0 \\
0.0 \\
.0 .0 \\
0.0 \\
0.0 \\
0.0 \\
0.0 \\
0.0 \\
0.0 \\
0.0 \\
0.0 \\
0.0 \\
0.0 \\
0.0 \\
0.0 \\
0.0 \\
0.0 \\
0.0\end{array}$ & $\begin{array}{l}0.0 \\
0.0 \\
0.0 \\
0.0 \\
0.0 \\
0.0 \\
0.0 \\
0.0 \\
0.0 \\
0.0 \\
0.0 \\
0.0 \\
0.0 \\
0.0 \\
0.0 \\
0.0 \\
0.0 \\
0.0 \\
0.0\end{array}$ \\
\hline
\end{tabular}


Table 3. (contd)

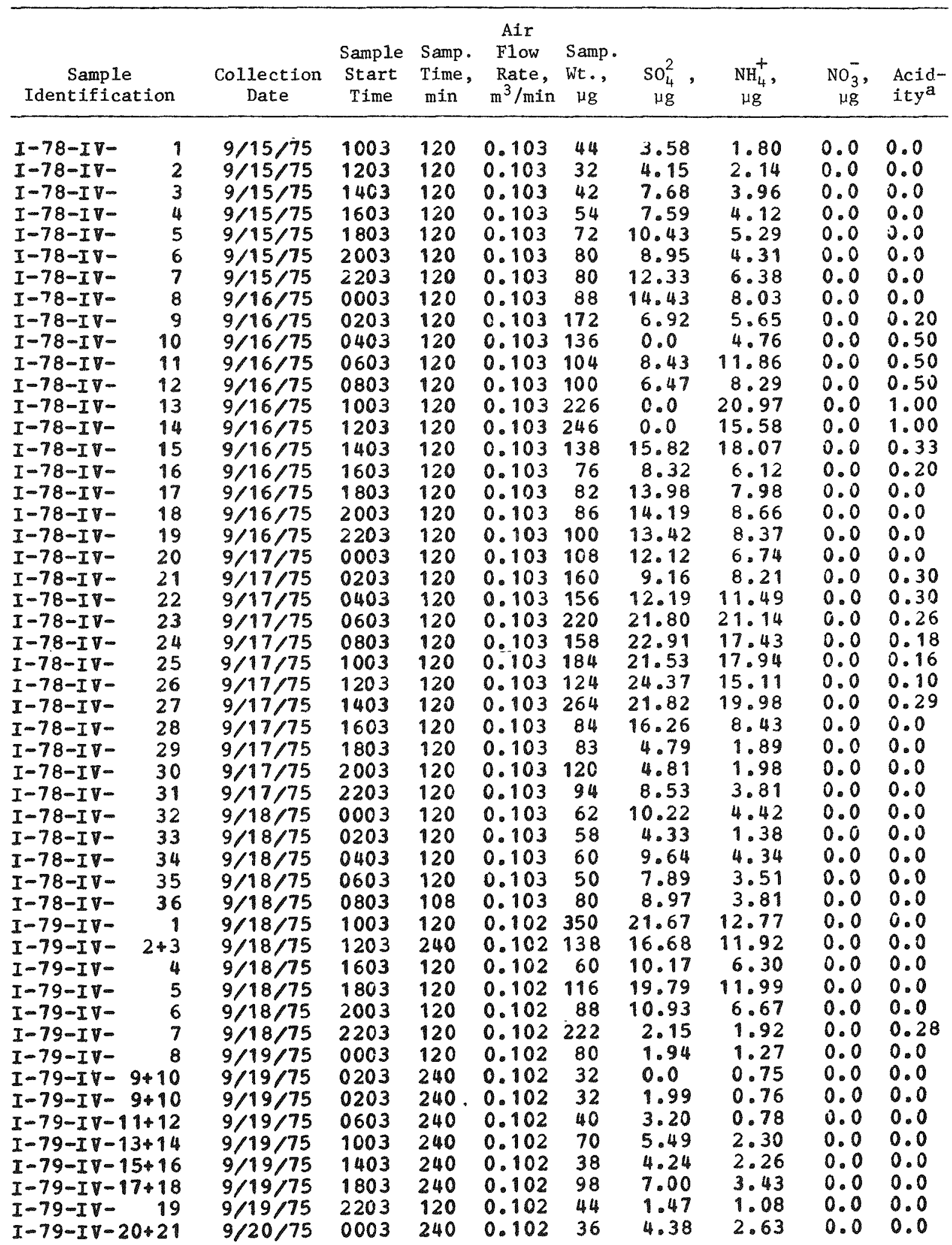


Table 3. (contd)

\begin{tabular}{|c|c|c|c|c|c|c|c|c|c|}
\hline $\begin{array}{c}\text { Sample } \\
\text { Identification }\end{array}$ & $\begin{array}{l}\text { 1lection } \\
\text { Date }\end{array}$ & $\begin{array}{c}\text { Sample } \\
\text { Start } \\
\text { Time }\end{array}$ & $\begin{array}{l}\text { Samp. } \\
\text { Time, } \\
\text { min }\end{array}$ & $\begin{array}{l}\text { Air } \\
\text { Flow } \\
\text { Rate, } \\
\mathrm{m}^{3} / \mathrm{min}\end{array}$ & $\begin{array}{l}\text { Samp. } \\
\text { Wt. } \\
\mu g\end{array}$ & $\begin{array}{c}\mathrm{SO}_{4}^{2-}, \\
\mu \mathrm{g}\end{array}$ & $\begin{array}{l}\mathrm{NH}_{4}^{+}, \\
\mu \mathrm{g}\end{array}$ & $\begin{array}{l}\mathrm{NO}_{3}^{-} \\
\mu \mathrm{g}\end{array}$ & $\begin{array}{l}\text { Acid- } \\
\text { itya }\end{array}$ \\
\hline $\begin{array}{lr}I-79-I V-22+23 \\
I-79-I V-24+25 \\
I-79-I V-26+27 \\
I-79-I V-28+29 \\
I-79-I V-30+31 \\
I-79-I V-32+33 \\
I-79-I V-34+35 \\
I-79-I V-\quad 36 \\
I-80-I V-\quad 1+2 \\
I-80-I V-\quad 3+4 \\
I-80-I V-\quad 5+6 \\
I-80-I V- & 7 \\
I-80-I V- & 8 \\
I-80-I V- & 9 \\
I-80-I V- & 10 \\
I-80-I V- & 11 \\
I-80-I V- & 12 \\
I-80-I V-13+14 \\
I-80-I V-15+16 \\
I-80-I V-17+18 \\
I-80-I V-19+20 \\
I-80-I V- & 21 \\
I-80-I V- & 22 \\
I-80-I V- & 23 \\
I-80-I V-24+25 \\
I-80-I V-26+27 \\
I-80-I V-28+29 \\
I-80-I V-30+31 \\
I-80-I V- & 32 \\
I-80-I V- & 33 \\
I-80-I V- & 34 \\
I-80-I V- & 35 \\
I-80-I V- & 36 \\
I-81-I V- & 5+6 \\
I-81-I V- & 7+8 \\
I-81-I V-9+10 \\
I-81-I V-11+12 \\
I-81-I V-13+14 \\
I-81-I V-15+16 \\
I-81-I V-17+18 \\
I-81-I V-19+20 \\
I-81-I V-21+22 \\
I-81-I V- & 23 \\
I-81-I V-24+25\end{array}$ & $\begin{array}{l}9 / 20 / 75 \\
9 / 20 / 75 \\
9 / 20 / 75 \\
9 / 20 / 75 \\
9 / 20 / 75 \\
9 / 21 / 75 \\
9 / 21 / 75 \\
9 / 21 / 75 \\
9 / 21 / 75 \\
9 / 21 / 75 \\
9 / 21 / 75 \\
9 / 21 / 75 \\
9 / 22 / 75 \\
9 / 22 / 75 \\
9 / 22 / 75 \\
9 / 22 / 75 \\
9 / 22 / 75 \\
9 / 22 / 75 \\
9 / 22 / 75 \\
9 / 22 / 75 \\
9 / 22 / 75 \\
9 / 23 / 75 \\
9 / 23 / 75 \\
9 / 23 / 75 \\
9 / 23 / 75 \\
9 / 23 / 75 \\
9 / 23 / 75 \\
9 / 23 / 75 \\
9 / 24 / 75 \\
9 / 24 / 75 \\
9 / 24 / 75 \\
9 / 24 / 75 \\
9 / 24 / 75 \\
9 / 24 / 75 \\
9 / 24 / 75 \\
9 / 25 / 75 \\
9 / 25 / 75 \\
9 / 25 / 75 \\
9 / 25 / 75 \\
9 / 25 / 75 \\
9 / 25 / 75 \\
9 / 26 / 75 \\
9 / 26 / 75 \\
9 / 26 / 75\end{array}$ & $\begin{array}{l}0403 \\
0803 \\
1203 \\
1603 \\
2003 \\
0003 \\
0403 \\
0803 \\
1004 \\
1404 \\
1804 \\
2204 \\
0004 \\
0204 \\
0404 \\
0604 \\
0804 \\
1004 \\
1404 \\
1804 \\
2204 \\
0204 \\
0404 \\
0604 \\
0804 \\
1204 \\
1604 \\
2004 \\
0004 \\
0204 \\
0404 \\
0604 \\
0804 \\
1813 \\
2213 \\
0213 \\
0613 \\
1013 \\
1413 \\
1813 \\
2213 \\
0213 \\
0613 \\
0813\end{array}$ & $\begin{array}{l}240 \\
240 \\
240 \\
240 \\
240 \\
240 \\
240 \\
109 \\
240 \\
240 \\
240 \\
120 \\
120 \\
120 \\
120 \\
120 \\
120 \\
240 \\
240 \\
240 \\
240 \\
120 \\
120 \\
120 \\
240 \\
240 \\
240 \\
240 \\
120 \\
120 \\
120 \\
120 \\
109 \\
240 \\
240 \\
240 \\
240 \\
240 \\
240 \\
240 \\
240 \\
240 \\
120 \\
165\end{array}$ & $\begin{array}{l}0.102 \\
0.102 \\
0.102 \\
0.102 \\
0.102 \\
0.102 \\
0.102 \\
0.102 \\
0.106 \\
0.106 \\
0.106 \\
0.106 \\
0.106 \\
0.106 \\
0.106 \\
0.106 \\
0.106 \\
0.106 \\
0.106 \\
0.106 \\
0.106 \\
0.106 \\
0.106 \\
0.106 \\
0.106 \\
0.106 \\
0.106 \\
0.106 \\
0.106 \\
0.106 \\
0.106 \\
0.106 \\
0.106 \\
0.105 \\
0.105 \\
0.105 \\
0.105 \\
0.105 \\
0.105 \\
0.105 \\
0.105 \\
0.105 \\
0.105 \\
0.105\end{array}$ & $\begin{array}{r}58 \\
30 \\
42 \\
22 \\
34 \\
36 \\
36 \\
26 \\
36 \\
56 \\
138 \\
60 \\
0 \\
48 \\
32 \\
136 \\
66 \\
61 \\
64 \\
88 \\
92 \\
72 \\
64 \\
76 \\
62 \\
30 \\
58 \\
76 \\
86 \\
94 \\
86 \\
86 \\
52 \\
12 \\
28 \\
58 \\
58 \\
48 \\
58 \\
32 \\
68 \\
0 \\
66 \\
40\end{array}$ & $\begin{array}{c}3.50 \\
2.99 \\
11.52 \\
3.95 \\
4.65 \\
0.0 \\
0.0 \\
2.83 \\
22.42 \\
22.35 \\
17.24 \\
16.70 \\
13.65 \\
11.39 \\
5.41 \\
0.0 \\
15.23 \\
11.72 \\
17.68 \\
27.53 \\
21.81 \\
24.66 \\
27.18 \\
21.35 \\
17.30 \\
7.14 \\
18.41 \\
15.80 \\
23.40 \\
18.16 \\
25.52 \\
16.69 \\
12.08 \\
4.97 \\
7.71 \\
12.37 \\
17.05 \\
19.06 \\
17.10 \\
9.91 \\
10.95 \\
10.49 \\
0.0 \\
0.0\end{array}$ & $\begin{array}{r}1.87 \\
0.75 \\
5.87 \\
2.54 \\
2.50 \\
20.96 \\
9.60 \\
4.07 \\
10.43 \\
12.95 \\
11.54 \\
9.82 \\
8.52 \\
7.40 \\
3.54 \\
5.10 \\
9.03 \\
6.93 \\
9.84 \\
14.84 \\
11.38 \\
12.83 \\
13.98 \\
10.86 \\
10.63 \\
4.47 \\
10.41 \\
9.57 \\
12.87 \\
10.26 \\
13.55 \\
10.22 \\
7.22 \\
2.97 \\
4.34 \\
7.02 \\
9.74 \\
10.51 \\
8.50 \\
6.01 \\
6.07 \\
6.58 \\
4.73 \\
3.49\end{array}$ & $\begin{array}{l}0.0 \\
0.0 \\
0.0 \\
0.0 \\
0.0 \\
0.0 \\
0.0 \\
0.0 \\
0.0 \\
0.0 \\
0.0 \\
0.0 \\
0.0 \\
0.0 \\
0.0 \\
0.0 \\
0.0 \\
0.0 \\
0.0 \\
0.0 \\
0.0 \\
0.0 \\
0.0 \\
0.0 \\
0.0 \\
0.0\end{array}$ & 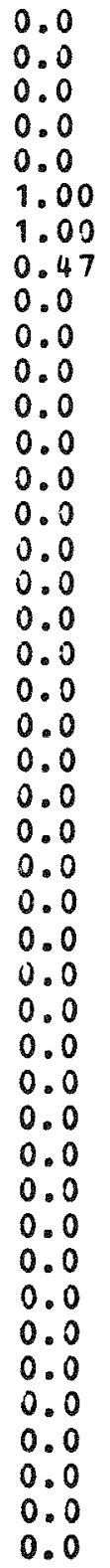 \\
\hline
\end{tabular}

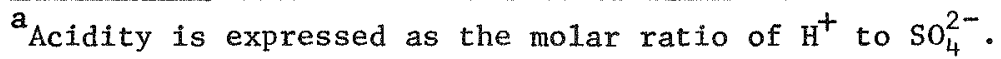




\section{DEVELOPMENT OF A NEW INSTRUMENT AND PROCEDURE}

\section{A. The ATR-Impactor Device}

A new approach for aerosol sampling and analysis, which combines the principle of impaction with attenuated total internal reflection (ATR) infrared spectroscopy, 6 has been developed. Such a device, called an ATRimpactor, could provide much greater sensitivity than the KBr-pellet technique described above, while minimizing manual sample handling; it also has the potential for fully automatic operation. A prototype unit has been designed and fabricated, and the initial laboratory and field tests have been completed. The design of the instrument and some of the initial results are presented in this section.

\section{Background}

The $\mathrm{KBr}$-pellet method has proven quite useful and continues to be employed on a routine basis in ongoing, large-scale studies of the chemistry of airborne particulate matter. However, as the scope of the studies has changed from single-site, short-term experiments to multisite, long-term programs which generate large numbers of samples, the need for an improved method for ambient aerosol sampling and analysis has become apparent.

Several aspects of the present $\mathrm{KBr}$-pellet method need improvement. First, sample preparation is time-consuming and tedious. The nature of the preparation procedure has defied attempts at mechanization and/or automation. With large-scale programs, the sample preparation makes an analysis of all of the samples prohibitively expensive. Another area where improvement is desirable is the time resolution of the sample, which is, of course, dependent on the analytical sensitivity. Also, in the present $\mathrm{KBr}$-pellet method, the possibility exists of altering the sample during collection and/or in the subsequent handling and time delay before analysis. For these reasons, an instrument is needed that would minimize sample handling, provide greater sensitivity, and approach real-time analysis. The ATR-impactor fulfills these needs.

\section{Design}

The ATR-impactor combines the use of an impactor for sample collection with ATR spectroscopy for sample analysis. A detailed discussion of the principles of ATR has been presented by Harrick. ${ }^{6}$ Briefly, ATR spectroscopy is based on the attenuation of infrared (IR) radiation as it is internally reflected at the outer surfaces of a suitable infrared-transparent medium. The medium is in the form of an internal reflection element (IRE), which has surfaces at appropriate angles so that the light may enter, reflect internally several times, and then exit to the detector. Light which has entered and is being transmitted through an IRE is attenuated by IR-absorbing substances which are within about one-half wavelength of, but external to, the surface of the IRE. Using the ATR principle, infrared absorption spectra of substances can be obtained by simply placing them in intimate contact with an IRE, as if the light had passed through the substances in the conventional way. The ATR spectrum closely resembles the absorption spectrum obtained by passing the light beam directly through the sample. By appropriately 
arranging the geometry of an IRE, multiple reflections can be achieved, each being comparable to a separate pass through the sample, resulting in greatly enhanced sensitivity.

It is possible to use the ATR principle for sensitive, real-time analysis of atmospheric aerosol by impacting particles directly onto an IRE arranged in an infrared spectrophotometer for combined sampling and analysis. This would be feasible only if the spectrophotometer or some other detection device can be operated in the field. Such a device would eliminate sample handling and preparation, while providing enhanced sensitivity and real-time analysis. However, in the prototype instrument that we have built, the spectrometer is not included in the sample-collection device. The sample is collected by impaction onto an IRE, which is subsequently transferred to the spectrometer without any additional sample preparation.

The currently used IREs are polished plates of KRS-5, which is $42 \mathrm{~mol} \%$ thallium bromide and $58 \mathrm{~mol} \%$ thallium iodide. KRS-5 is attractive for ATR work due to its high refractive index $\left(2.38\right.$ at $\left.2000 \mathrm{~cm}^{-1}\right)$ and its wide range of transmission (5000-250 $\mathrm{cm}^{-1}$ ) in the infrared region. However, KRS-5 is fairly soft, is toxic due to its thallium content, and must therefore be handled with care. Other materials, such as germanium and zinc selenide, are available and have also been used in the unit.

The prototype unit, shown schematically in Fig. 4, consists of four impactor stages; the first three are from a cascade centripeter--a commercially available virtual impactor (BGI, Inc., Waltham, Massachusetts). The centripeter stages have nominal $50 \%$ cut points of $14-, 4-$, and $1.2-\mu \mathrm{m}$ aerodynamic diameter, and are used to remove the larger particles which are not analyzed. The fourth stage has a nominal $50 \%$ cut-point value of $0.5 \mu \mathrm{m}$ and thus collects the accumulation-mode size fraction of the sampled ambient aeroso1. As shown in Fig. 4, the air flow is split after it passes the third stage of the centripeter and is then drawn through two separate nozzles so that the particles are deposited on the two opposite sides of the IREs or ATR plates. Time resolution is provided in the fourth stage by moving the IREs past the nozzles at a controlled rate. This is shown more clearly in Fig. 5, which shows detailed front and side views of the air nozzles and IREs in the ATR-impactor.

The fourth stage of the ATR-impactor was designed according to the procedure of Marple and Willeke. ${ }^{7}$ For an air flow rate of $30 \mathrm{~L} / \mathrm{min}$, which is the nominal operating air flow for a single centripeter, the specifications for the fourth stage nozzle are as follows: slit width, $0.203 \pm$ $0.025 \mathrm{~mm}$; slit length, $20.0 \pm 0.13 \mathrm{~mm}$; slit throat depth, $0.203+0.13 \mathrm{~mm}$; and slit-to-plate distance, $0.305 \pm 0.05 \mathrm{~mm}$. The collection surfaces are formed by the IRE, which is $25-\mathrm{mm}$ wide $\times 5-\mathrm{mm}$ long $\times 2-\mathrm{mm}$ thick. The ends of the IRE are beveled at an angle of $45^{\circ}$, as shown in the top view in Fig. 5. A second set of nozzles was constructed with slit widths of $0.406 \pm 0.025 \mathrm{~mm}$, for use with the higher flow rates needed for airborne plume studies. With these nozzles, the flow rate was increased to a nominal value of $60 \mathrm{~L} / \mathrm{min}$. Two centripeters mounted in parallel on a special headend adapter were used to accommodate this higher flow rate. Flow-limiting critical orifices were inserted between the impactor and the pump to control the flow. A differential pressure gauge was permanently mounted across the critical orifice to monitor the pressure drop across the orifice. 


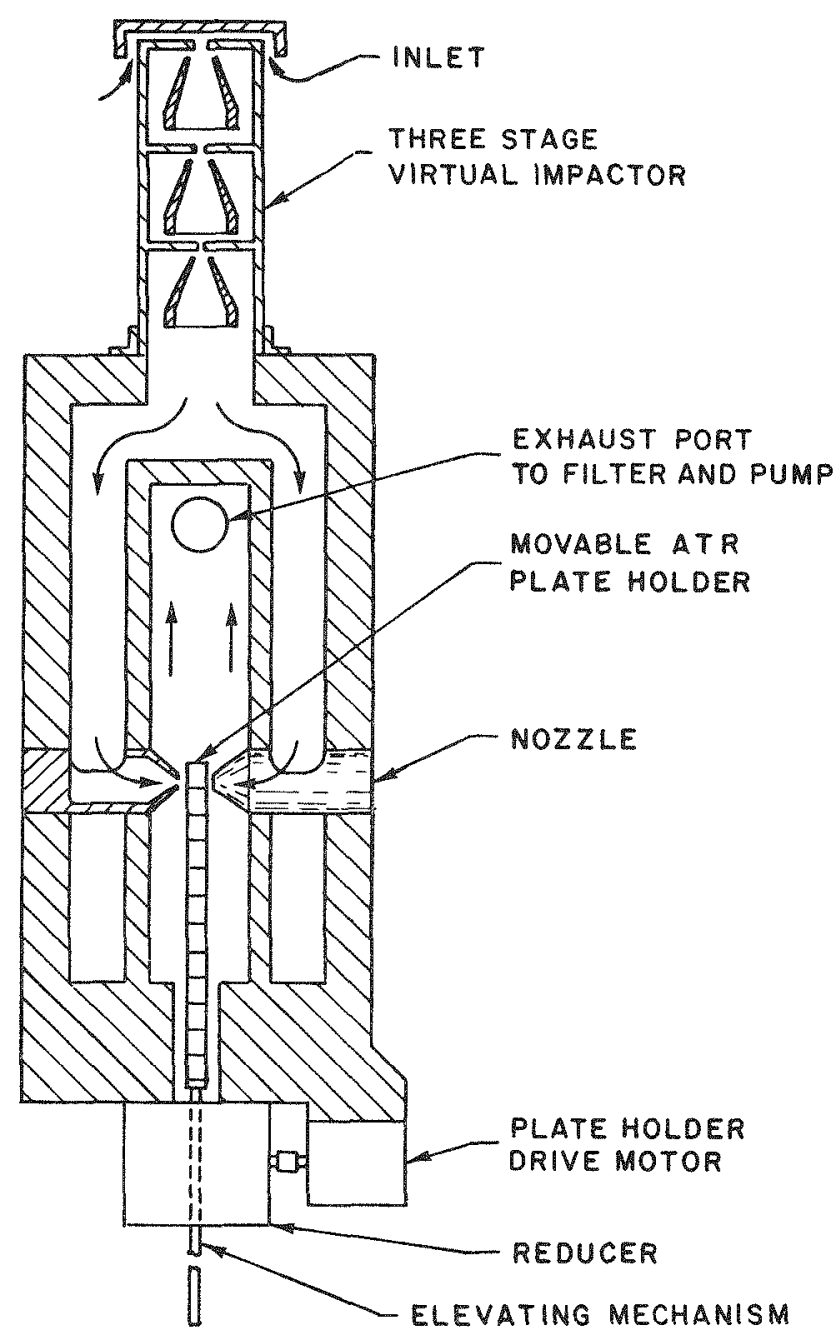

Fig. 4. Schematic Diagram of the ATR-Impactor

In the present design, the ATR-impactor system is not fully automated. The IREs with the collected samples are removed from the impactor for analysis on a Digilab Mode1 FTS-14 Fourier-transform infrared (FTIR) spectrophotometer. A 4X variable-angle beam condenser, Mode1 4XTBC-VA (Harrick Scientific Corp., Ossing, New York) is used on the IR spectrophotometer to accommodate the IREs. In the prototype unit, up to 20 individual IRE plates are held side by side in a holder. During collection, the holder is driven at a constant speed, thereby moving the series of IREs between the two nozzles and collecting an even deposit of particles on the opposite surfaces. During analysis, an individual spectrum is obtained from each IRE to provide the time resolution. Collection times can be varied by changing the drive speed. 

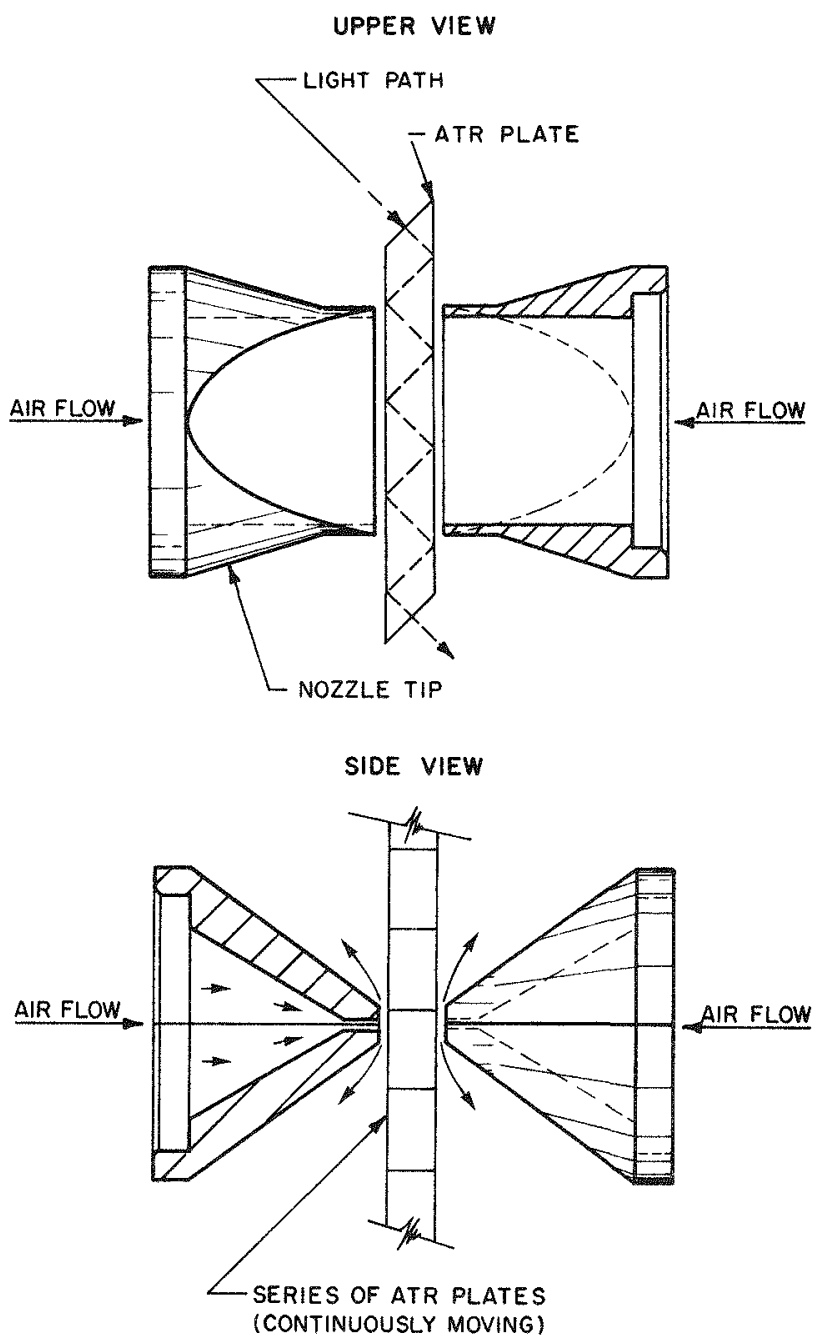

Fig. 5 .

Detail of Nozzles and ATR Plates

It is anticipated that future ATR-impactor units could have builtin infrared light sources and detectors, tuned to the appropriate wavelengths of 1ight. In this way, specific species could be detected as they are collected, thereby supplying near real-time detection. If necessary, a stream of inert gas could be passed over the ATR plates and samples immediately after collection to limit their exposure to any reactive ambient gases.

\section{Results and Discussion}

\section{a. Initial Field Trials}

In the first field trials of the ATR-impactor using the 30-L/min air flow nozzles, a comparison was made between the ATR method and our standard $\mathrm{KBr}$ method. Figure 6 presents infrared spectra of ambient samples collected simultaneously and analyzed using the two methods. As expected, for the submicrometer-sized ambient particulate matter, the spectra reveal the presence of ammonium sulfate and nitrate. To make the spectra directly comparable, the absorbance scale has been adjusted to account for differences in the air sampling rate. The ATR spectrum was from a sample 


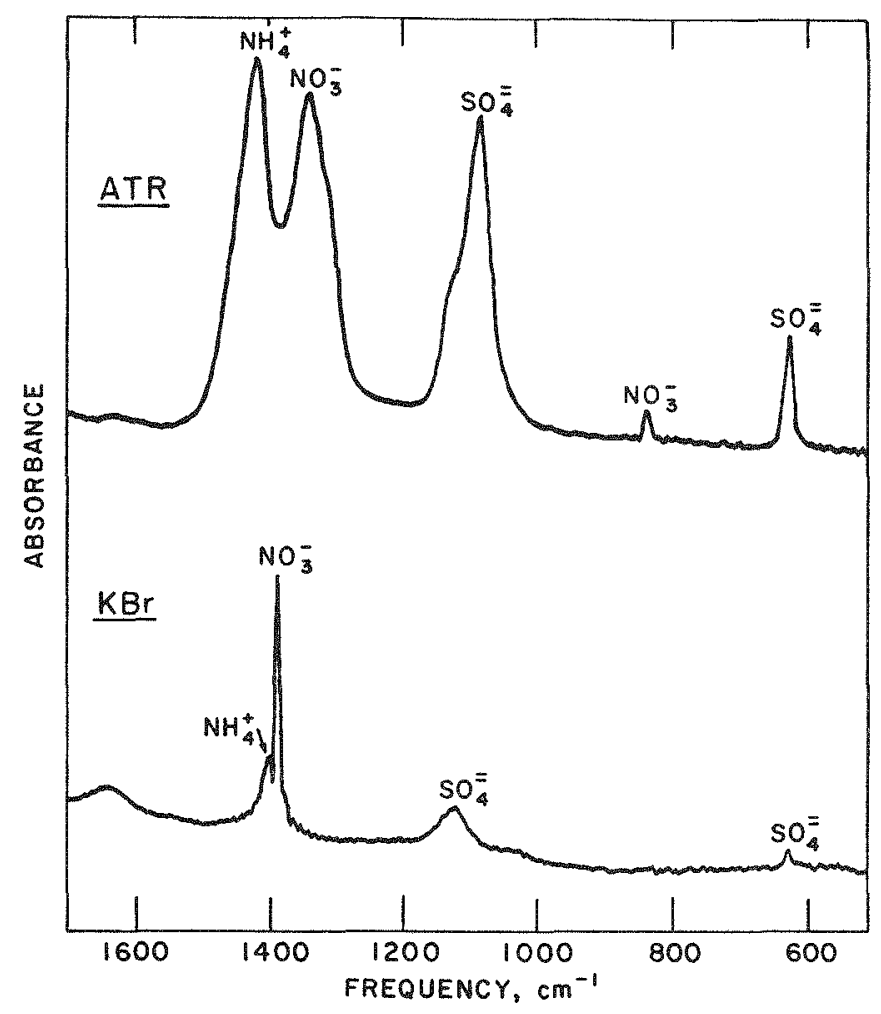

Fig. 6. Infrared Spectra of Ambient Aerosol Samples Collected Simultaneously to Illustrate the Greater Sensitivity of the ATR Method vs. the KBrPellet Method

collected for $55 \mathrm{~min}$ at a flow rate of $30 \mathrm{~L} / \mathrm{min}$. Based on our established calibration curves for the $\mathrm{KBr}$ method using the $620-\mathrm{cm}^{-1}$ sulfate band, there were $n 10 \mu \mathrm{g}$ of sulfate on the IRE. The absorbance for the $620-\mathrm{cm}^{-1}$ band in the ATR spectrum is 0.14 absorbance units. As can be seen in Fig. 6, the absorbance at $620 \mathrm{~cm}^{-1}$, based on peak height, is at least six times greater in the ATR spectrum compared to the $\mathrm{KBr}$ spectrum which represents the same amount of sulfate. Similarly, the $1110-\mathrm{cm}^{-1}$ sulfate band, the $1400-\mathrm{cm}^{-1}$ ammonium band, and the $840-\mathrm{cm}^{-1}$ nitrate band are comparably enhanced in the ATR spectrum.

A different situation exists for the $1384-\mathrm{cm}^{-1}$ nitrate band and warrants further explanation. It has recently been found that this very sharp and strong band at $1384 \mathrm{~cm}^{-1}$ is due to a matrix effect of the $\mathrm{KBr}$ on the nitrate and not to a chemisorbed or surface nitrate species as previously believed. The matrix effect causes the nitrate to appear as a free ion. Because of its strength, the $1384-\mathrm{cm}^{-1}$ band is a very sensitive indicator of the nitrate content of samples prepared in $\mathrm{KBr}$ pellets. In the ATR spectrum, this nitrate band is much broader and occurs at $1337 \mathrm{~cm}^{-1}$ since the matrix effect is absent on the IRE. Thus, for this nitrate band, the ATR method is only slightly more sensitive than the $\mathrm{KBr}$ method. The $840-\mathrm{cm}^{-1}$ nitrate band 
is seen in the ATR spectrum but is too weak to be detected in the $\mathrm{KBr}$ spectrum since the matrix does not enhance this nitrate absorbance mode.

It should be mentioned that the bands are shifted by varying degrees in the two types of spectra. Only small shifts are seen for most bands, except, as explained above, for the $1384-\mathrm{cm}^{-1}$ nitrate and the $1110-\mathrm{cm}^{-1}$ sulfate bands which are both noticeably shifted. Laboratory studies of known materials have verified the identity of these shifted bands. The shoulder at $1120 \mathrm{~cm}^{-1}$ in the ATR spectrum has not been identified, but, as will be seen, it may be related to the nitrate bands.

In Fig. 7, two ATR spectra of the same ambient sample are shown. The lower spectrum, labeled fresh, was obtained within ten minutes after collection. Additional spectra were obtained from the sample within 24 hours following collection and no changes were detected. However, the top spectrum, labeled aged, was obtained after six-months storage and, as can be seen, several changes had occurred. The nitrate bands at $1337 \mathrm{~cm}^{-1}$ and $830 \mathrm{~cm}^{-1}$ had disappeared, the $1415-\mathrm{cm}^{-1}$ ammonium band had diminished, and the $1120-\mathrm{cm}^{-1}$ shoulder was barely detectable. Since the two sulfate bands at $1080 \mathrm{~cm}^{-1}$ and $615 \mathrm{~cm}^{-1}$ remained unchanged, this indicated that the ammonium nitrate in the sample was volatile enough to be completely removed during the storage period. The sample had been stored on the KRS-5 IRE in air in a closed plastic Petri dish. Unfortunately, no other spectra were obtained during the storage period.

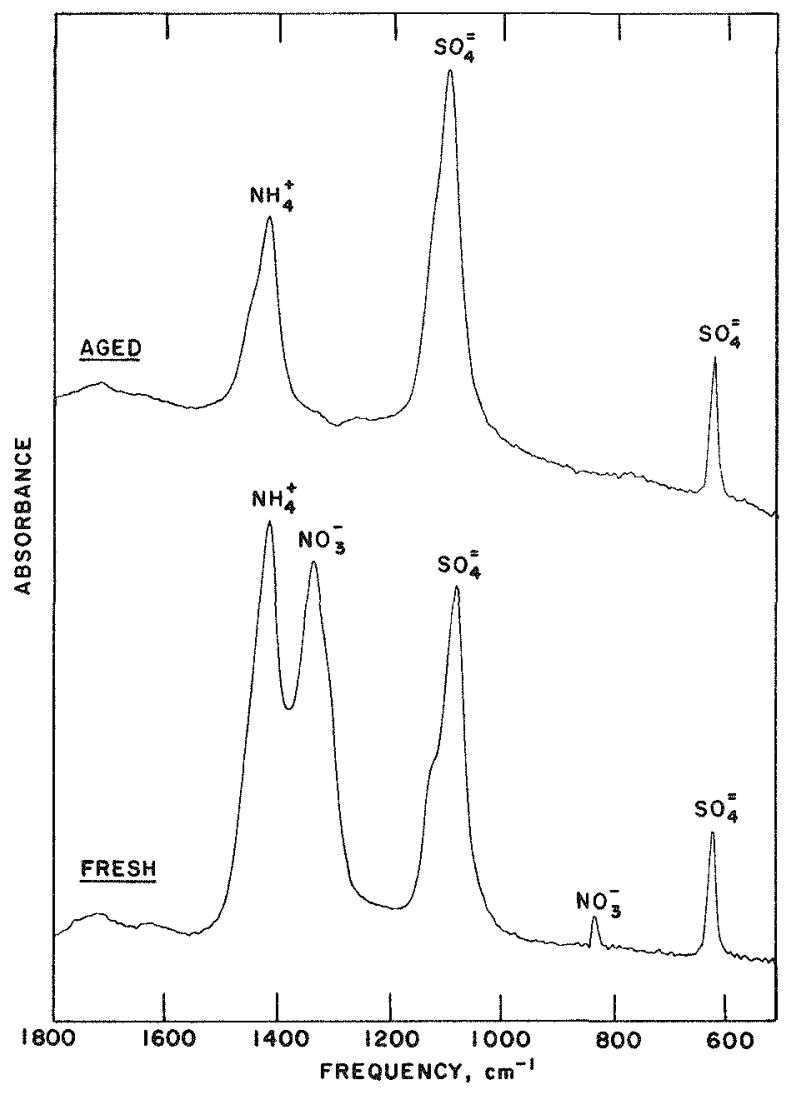

Fig. 7.

Infrared Spectra of an Ambient Aerosol Sample Immediately after Collection (fresh) and after Six-Months Storage (aged) 
One of the objectives in the design of the ATR-impactor was to supply time resolution short enough to enable the study of the changing aerosol chemistry of a power-plant plume. In preparation for the airborneplume studies, ground-based tests were conducted at ANL using the dual centripeter inlet and a $75 \mathrm{~L} / \mathrm{min}$ air flow rate. Samples were collected with 12-min, 4-min, and 1-min time resolution. The spectra of all of these samples revealed the presence of neutral ammonium sulfate. Figure 8 presents the spectra from the 4-min and the 1-min samples. As is evident, even in the 1-min sample the ammonium sulfate absorptions are easily detectable. It is estimated from a sample collected with another impactor and analyzed in the routine manner using a $\mathrm{KBr}$ pellet that the 1-min sample contained $\sim 0.5 \mu \mathrm{g}$ of sulfate.

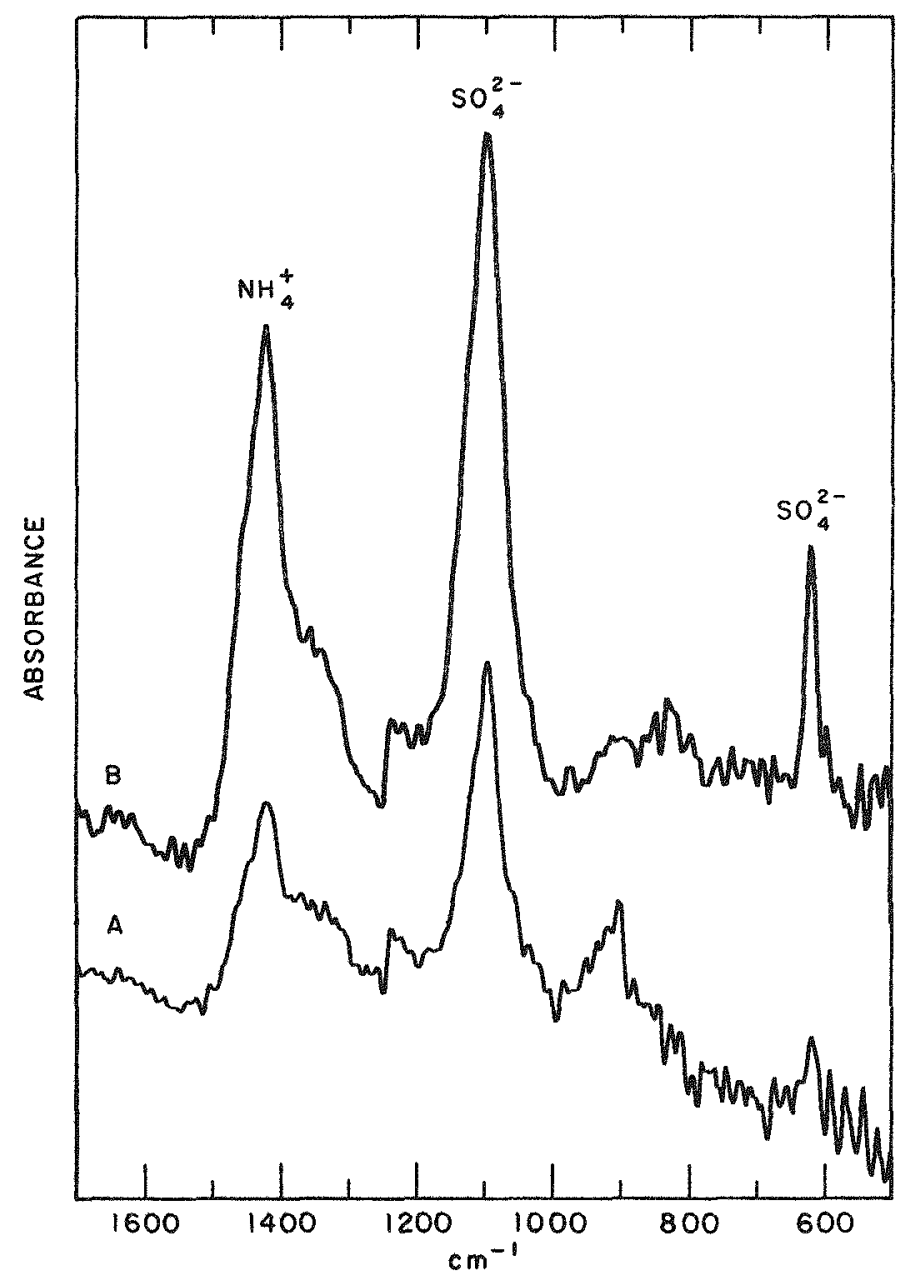

Fig. 8. Infrared Spectra of Submicrometer Ambient Aerosol Collected with ATR-Impactor. A: 1 minute collection at $75 \mathrm{~L} / \mathrm{min}$. $\mathrm{B}$ : 4 minute collection at $75 \mathrm{~L} / \mathrm{min}$. 


\section{b. Airborne-Plume Studies}

During the week of April 14-18, 1980, the ATR-impactor underwent airborne trials in a plume from a power plant near Centralia, WA. The trials were conducted using the Battelle Pacific. Northwest Laboratory (PNL) DC-3 aircraft which is fully instrumented for airborne pollutant studies. Our main objective for these trials was to demonstrate the feasibility of using the ATR-impactor for studies of plume chemistry and to determine what problems might arise. A constant flow rate of $64 \mathrm{~L} / \mathrm{min}$ was established using a calibrated critical orifice and a rotary vane vacuum pump (Mode1 0740, Gast Corp., Benton Harbor, Michigan) outfitted with a 28VDC motor. To facilitate the quick changes needed to accommodate the varying collection conditions during the flights, the unit was equipped with a reversible, variable-speed control unit (Mode1 IMI00, Minarik Electric Co., Los Angeles, CA) for driving the internal reflection elements. This arrangement worked very well.

Figure 9 presents three of the ATR spectra from samples collected during the flights. As can be seen in Fig. 9, sulfate can be

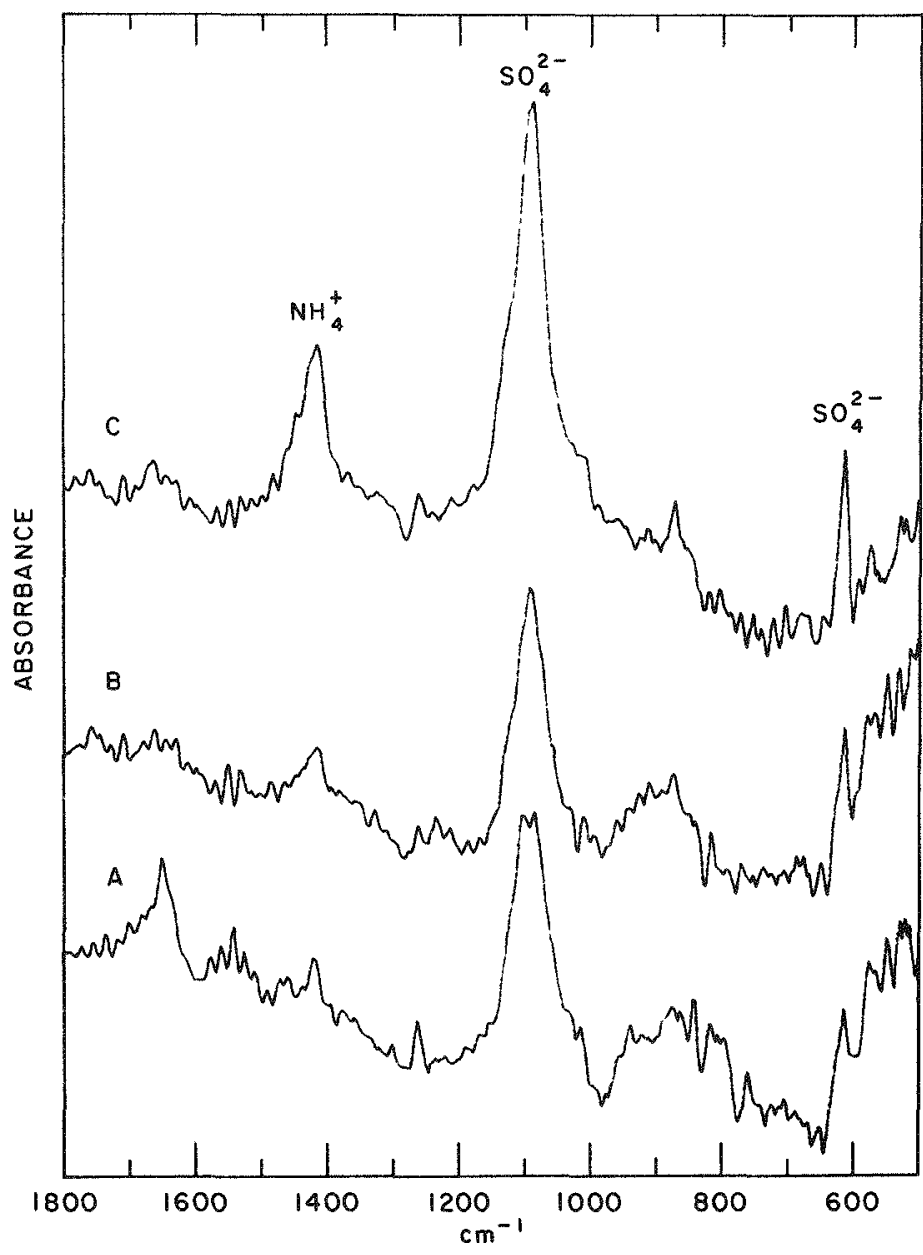

A: SINGLE TRAVERSE, 6 MILES FROM STACK.

$B$ : SINGLE TRAVERSE, 20 MILES FROM STACK.

C: THREE CONSECUTIVE TRAVERSES, 20 MILES FROM STACK.
Fig. 9.

Infrared Spectra of PowerPlant Plume Samples Collected with ATR-Impactor 
easily detected even in a single traverse of the plume with a collection time of 101 seconds. It was estimated that the total plume particle loading for this sampling was about $10 \mu \mathrm{g} / \mathrm{m}^{3}$, which would correspond to a fairly typical loading of only sulfate for summertime ambient air in the Midwest.

\section{c. General Observations}

Some general observations have been made during the initial work with the ATR-impactor. Microscopic examination of the sample on the IRE revealed that for a one-hour collection period, where $\sim 10 \mu \mathrm{g}$ of sulfate was collected, less than $25 \%$ of the surface of the IRE was actually covered with the particulate matter. With this small amount of coverage, each individual particle has open area between it and the nearest neighbor. This is important in minimizing interactions between collected particles. Spectra obtained from ambient samples with 15-min time resolution (15-min per IRE) showed that the coverage of the IRE surface was below $10 \%$. For a 1-min sampling, the coverage would be even less. The limit of detection is estimated to be we11 below $0.5 \mu \mathrm{g}$ of sulfate per sample.

In addition to the field tests of the ATR-impactor, some laboratory work has also been done. This involved the collection and analysis of aerosols generated from known materials, mainly for the purposes of IR absorption band verification.

\section{Summary}

The ATR-impactor has successfully completed initial laboratory and field tests and has shown that it offers several important advantages for the spectroscopic chemical characterization of ambient and plume aerosol. It has been shown that the infrared bands of the collected material are at least six times more intense than the bands obtained by the conventional $\mathrm{KBr}$-pellet technique. For sulfate, the limit of detection is thus less than $0.5 \mu \mathrm{g}$. This is sufficient for 1 -min time resolution on the analysis of typical ambient concentrations of sulfate using the prototype unit with a flow rate of $64 \mathrm{~L} / \mathrm{min}$. Time resolution of this order is desirable for plume studies, as well as for detailed studies which may lead to a better understanding of the dynamics of atmospheric chemistry.

Another advantage lies in the virtual elimination of the timeconsuming sample handling and preparation procedures. This enables the incorporation of spectral measurement devices directly into the sampling unit, thereby providing for analysis as the sample is being collected.

\section{B. X-Ray Fluorescence Method for the Determination of Proton Acidity in Aerosol Sulfates}

This new method is being developed with the objective of measuring the proton acidity of atmospheric aerosol samples directly on the collection substrate by means of an acid-specific reagent of suitable characteristics. The important properties of such a proton-acceptor reagent include volatility, selectivity, stability, and detectability. The possibility of using a proton acceptor containing a heavy-element tag that could be detected by X-ray fluorescence spectroscopy (XRF) was investigated because of the potential for 
good selectivity and sensitivity provided by the XRF technique. In addition, such a method would be well suited for automated analyses and facile introduction into existing monitoring programs.

In the proposed method, the atmospheric aerosol sample would be exposed to a vapor-phase, tagged, proton acceptor until any acidity in the sample is completely neutralized. The excess reagent would be removed by flushing with an inert gas, leaving behind a nonvolatile tagged neutralization product. The amount of the tag element would then be measured by the XRF technique, and the acidity of the aerosol sample would thus be determined.

\section{Reagent Selection}

The characteristics which the reagent of choice should exhibit are:

(a) Volatility: The proton acceptor should be volatile so that reaction with the sample could be carried out in the gas phase to prevent undesired reactions or losses resulting from solvent effects. In addition, excess reagent could be removed by gentle heating under an inert gas flow. It is assumed that the product of the reaction would be a nonvolatile compound which would become immobilized on the sample collection substrate.

(b) Selectivity: The reagent of choice should be specific for the strong acid components in the sample, such as $\mathrm{H}_{2} \mathrm{SO}_{4}$ and $\mathrm{HSO}_{4}^{-}$, and should not react with such weak acids as water vapor or ammonium ion, which are expected to be present in the sample.

(c) Stability: The reagent of choice should be resistant to decomposition by air oxidation or high temperatures.

(d) Detectability: The reagent of choice must contain a functional group which may be determined quantitatively at levels corresponding to sample acid concentrations by means of a selective, sensitive method. Possible groups which have been suggested include radioactive "tags" such as tritium or carbon-14, fluorescent organic functional groups, and heavyelement tags suitable for determination by $X$-ray fluorescence. $X$-ray fluorescence is already used for routine elemental analysis of filter samples and possesses outstanding selectivity and sensitivity characteristics. Acidity measurements by this method could thus be readily introduced into existing monitoring programs.

Examination of possible candidates for the proton-acceptor reagent led to the choice of an organo-germanium compound containing an amino group on one of the organic side chains. Gas-phase titrations of acid aerosols and particulate matter using ammonia have been described, and a method for sulfuric acid using diethyl amine has been used to determine total sulfate acidity. 8 Organo-germanium compounds show excellent stability, and the sensitivity of the XRF technique for the determination of germanium is exceptional.

Because of the above considerations, as well as ease of reagent synthesis, trimethy 1-3-aminopropy1-germane $\left[\left(\mathrm{CH}_{3}\right)_{3} \mathrm{Ge}-\left(\mathrm{CH}_{2}\right)_{3}-\mathrm{NH}_{2}\right]$, TAPG, was selected as the reagent for investigating the feasibility of this technique. 


\section{Discussion of Experimental Procedures}

\section{a. Impactor Samples}

A flanged glass neutralization chamber was used in the experiments. The samples were placed in this chamber which was then flushed with dry nitrogen. The amine was injected into the chamber and the reaction allowed to proceed at a controlled temperature for a predetermined length of time. Determination of the germanium content of the sample was performed on a General Electric Company XRD- 6 wavelength-dispersive XRF instrument. The Ge-K $\alpha$ line at $36.33^{\circ}(2 \theta)$ from a LiF crystal was used as the analytical line.

Initially, laboratory-synthesized samples were used in the development of this procedure. These samples were prepared by placing 2 to $10 \mu \mathrm{L}$ of solutions containing $\sim 10 \mu \mathrm{g} / \mu \mathrm{L} \mathrm{H}_{2} \mathrm{SO}_{4}$ or $\mathrm{NH}_{4} \mathrm{HSO}_{4}$, or $\sim 50 \mu \mathrm{g} / \mu \mathrm{L}\left(\mathrm{NH}_{4}\right)_{2} \mathrm{SO}_{4}$ on Mylar or filter substrates.

The reagent, trimethy 1-3-aminopropy1-germane, proved to be a good choice. It does not decompose at its normal boiling point of $157^{\circ} \mathrm{C}$. After several months of working with the compound, there was no indication of sensitivity to air, light, or temperature. After a large number of cycles ( 100$)$ involving the introduction, vaporization, and flushing of the amine in the neutralization chamber, no visible signs of a residue were observed. However, one of the difficulties encountered with the reagent was its low vapor pressure. It is estimated that, at room temperature, the reagent's vapor pressure is $\sim 3$ to 4 torr. This increases to $\sim 20$ torr at $50^{\circ} \mathrm{C}$ and $\sim 150$ torr at $100^{\circ} \mathrm{C}$.

The first phase of the work was devoted to finding a suitable set of operating conditions for the neutralization process. The primary requirements are quantitative reaction with the strongly acidic protons in $\mathrm{H}_{2} \mathrm{SO}_{4}$ and $\mathrm{NH}_{4} \mathrm{HSO}_{4}$, and minimum response to $\left(\mathrm{NH}_{4}\right)_{2} \mathrm{SO}_{4}$ and other species in atmospheric samples.

At room temperature and 1 -atm pressure, the reaction time required for the neutralization was very long. Even after 30 minutes, unacceptably low recoveries were obtained for the prepared samples. Upon partial evacuation of the neutralization chamber (to increase the rate of reagent mass transfer), good recoveries were obtained for $\mathrm{H}_{2} \mathrm{SO}_{4}$ with a reaction time of 15 minutes. However, the recoveries for $\mathrm{NH}_{4} \mathrm{HSO}_{4}$ were erratic and ranged from 30 to $70 \%$. Response to $\left(\mathrm{NH}_{4}\right)_{2} \mathrm{SO}_{4}$ was generally low (equivalent to $10 \%$ or less of the $\mathrm{NH}_{4}^{+}$ion) but was as much as 60 to $100 \%$ at times. This sporadic behavior is not completely understood, but the primary cause appeared to be insufficient drying of the sample before introduction of the amine into the neutralization chamber.

It was hypothesized that residual water in the sample acted as a proton transfer agent allowing an exchange of the organic amine for ammonia which is a weaker base in aqueous solution. An experiment was carried out to test this hypothesis. In this experiment, several samples of ammonium bisulfate were prepared and neutralized with TAPG without drying. The reaction with ammonium ion was quantitative under these conditions as indicated by an average germanium recovery of $202 \%$ relative to the acid expected in the samples. 
This result demonstrated a need for a reliable method of drying the samples prior to neutralization. Oven drying of synthetic samples was tested and gave good results from the standpoint of reducing reaction with ammonium ion, but low recoveries of $\mathrm{H}_{2} \mathrm{SO}_{4}$ were obtained even for low oven temperatures $\left(40^{\circ} \mathrm{C}\right)$. Often, low $\mathrm{H}_{2} \mathrm{SO}_{4}$ recovery was accompanied by strong discoloration of the substrate. We believe that the $\mathrm{H}_{2} \mathrm{SO}_{4}$ losses were due to either volatilization or reaction of the sulfuric acid during the drying step. After some experimentation, it was determined that storing the samples overnight in a desiccator charged with silica gel would result in complete drying without loss of $\mathrm{H}_{2} \mathrm{SO}_{4}$.

A series of synthetic standards dried in this manner were neutralized with TAPG to better define the neutralization conditions. For these tests, the samples were placed in the neutralization chamber and TAPG $(\sim 100 \mu \mathrm{L})$ was injected into the chamber. All stopcocks on the chamber were tightly closed and it was placed in a drying oven at a preset temperature for 30 minutes. After this time, the chamber was removed from the oven to a laboratory hood and flushed with dry air (dried over molecular sieves) to remove the excess reagent. For efficient removal, a heat lamp illuminated the chamber while it was flushed out. After approximately 15 minutes flushing time, the samples were removed, fixed with a drop of collodion in ethyl acetate and subjected to XRF analysis.

Using this procedure, average recoveries for several series of synthetic samples were determined using neutralization temperatures of $89^{\circ} \mathrm{C}$ and $50^{\circ} \mathrm{C}$. The results were:

\begin{tabular}{|c|c|c|c|}
\hline \multirow[b]{2}{*}{ Temperature } & \multicolumn{2}{|c|}{ Recovery of Acid by XR } & \multirow[b]{2}{*}{$\left(\mathrm{NH}_{4}\right)_{2} \mathrm{SO}_{4}$} \\
\hline & $\mathrm{H}_{2} \mathrm{SO}_{4}$ & $\mathrm{NH}_{4} \mathrm{HSO}_{4}$ & \\
\hline $89^{\circ} \mathrm{C}$ & $64 \%$ & $101 \%$ & $2.7 \%$ \\
\hline $50^{\circ} \mathrm{C}$ & $92 \%$ & $100 \%$ & $1.0 \%)$ \\
\hline
\end{tabular}

The low recovery of $\mathrm{H}_{2} \mathrm{SO}_{4}$ at the higher temperature is probably due to losses of the acid by volatilization or reaction prior to its neutralization by the TAPG reagent. A neutralization temperature of $50^{\circ} \mathrm{C}$ was used in all subsequent experiments. Since the results demonstrated that a usable procedure for synthetic samples had been obtained, work was begun to evaluate the method's performance with field-type samples.

The field samples used for this evaluation were archive samples of sulfate aerosol collected on Mylar with a Lundgren impactor as part of another program. Acidic samples were desired for the study, so samples were selected from a time period corresponding to an acidic episode as indicated by FTIR analysis. The samples were in the form of a line of impacted material $40-\operatorname{mm}$ long on Mylar strips $\sim 3-\mathrm{mm}$ wide. For each sample used, $\sim 15 \mathrm{~mm}$ was taken for FTIR analysis which provided a value for the total sulfate (as micromoles $\mathrm{SO}_{4}^{2-}$ ), as well as the relative acidity of the sulfate (e.g., more acidic or less acidic than bisulfate). The remaining sample was divided into several sections which were used for determination of the proton acidity by neutralization with the TAPG reagent or by measuring the $\mathrm{pH}$ change 
of a weakly acidic ( $\mathrm{pH}$ 4.5) leach solution upon addition of the sample. This reference $\mathrm{pH}$ method for proton acidity was shown to be reliable for strong-acid synthetic samples $\left(\mathrm{H}_{2} \mathrm{SO}_{4}\right.$ and $\left.\mathrm{NH}_{4} \mathrm{HSO}_{4}\right)$ deposited on Mylar.

The data obtained through the various methods for the field samples are summarized in Table 4. In order to simplify comparison of the data, the values in Table 4 are generally given in terms of micromoles or microequivalents per millimeter of sample. This was done because the samplesize requirements differ among the methods so that varying sample lengths had to be used.

Table 4. Summary of Data from Analysis of Field Samples of Sulfate Aerosol

\begin{tabular}{|c|c|c|c|}
\hline \multirow{3}{*}{$\begin{array}{c}\text { Sample } \\
\text { Identification }\end{array}$} & \multicolumn{3}{|c|}{ Method } \\
\hline & FTIR & $\Delta \mathrm{pH}$ & $\mathrm{XRF}-\mathrm{Ge}$ \\
\hline & $\mu \mathrm{mol} \mathrm{SO}_{4}^{2-} / \mathrm{mm}$ & Leq $\mathrm{H}^{+} / \mathrm{mm}$ & heq $\mathrm{H}^{+} / \mathrm{mm}$ \\
\hline \multirow[t]{2}{*}{ IPS-102-IV-15 } & 0.0335 & 0.0332 & 0.0318 \\
\hline & & & 0.0352 \\
\hline $\begin{array}{l}\text { IPS-102 After- } \\
\text { Filter }\end{array}$ & $\begin{array}{c}\text { None } \\
\text { Detectable }\end{array}$ & $<0.0003\left(\operatorname{per} \mathrm{mm}^{2}\right)$ & $<0.0003\left(\right.$ per $\left.\mathrm{mm}^{2}\right)$ \\
\hline \multirow[t]{2}{*}{ IPS-102-IV-14 } & 0.0268 & 0.0307 & 0.0308 \\
\hline & & 0.0260 & 0.0602 \\
\hline \multirow[t]{4}{*}{ IPS-102-IV-13 } & 0.0305 & 0.0269 & 0.0860 \\
\hline & & & 0.0412 \\
\hline & & & 0.0492 \\
\hline & & & 0.0451 \\
\hline \multirow[t]{3}{*}{ IPS-102-IV-12 } & 0.0178 & -- & 0.0526 \\
\hline & & & 0.0349 \\
\hline & & & 0.0538 \\
\hline
\end{tabular}

The FTIR results indicated that the sulfate acidity of all the samples was near that of bisulfate. This observation is supported by the close correspondence between the microequivalents of acid found by $\mathrm{pH}$ measurement and the micromoles sulfate by FTIR. Some caution is advised in comparing these results too closely, however, for two reasons. First, the precision of either method at this range of sample size is not better than $15 \%$. Second, some inhomogeneity or unevenness exists in the distribution of the sample on the Mylar substrate, as evidenced by microscopic examination of the samples and the failure of different sections of the sample to give duplicate results even within $15 \%$, 
Initial results from the XRF method were extremely encouraging. For the first field sample that was analyzed, IPS-102-IV-15, very good agreement among all three methods was obtained. Next, a sample from the impactor after-filter of IPS-102 was used, and values from al1 three methods fell below their respective limits of detection. This result was also interpreted as good agreement.

The second impactor sample, IPS-102-IV-14, gave mixed results. The values for pH measurement, from FTIR analysis, and one XRF result agreed satisfactorily. However, the other XRF result was high by a factor of about two. It appeared that sample inhomogeneity was affecting the data. Because all three methods employed here are destructive and it was not possible to reexamine the samples already run, a more carefully controlled experiment was planned for the next set of samples.

In this experiment, a permanent record of the appearance of the samples was first obtained by optical micrography. Examination of the photomicrographs showed:

(1) The impacted material was not evenly distributed along the length of the line on the substrate.

(2) Material on the Mylar substrate was not restricted to the vicinity of the line; a thin layer of wet-looking material coated the Mylar to varying widths, sometimes reaching to the edges of the Mylar strip.

(3) Droplets and smears of a viscous, colorless liquid were visible over broad areas of the sample. The distribution of these droplets was often localized and by no means homogeneous.

Once they were photographed, the samples were placed to dry in a desiccator over silica gel over the weekend. Surprisingly, drying in this way changed the sample's microscopic appearance very little. In particular, the size and location of the liquid droplets remained the same. The Mylar strip was then divided into several sections for analysis. Neutralization with TAPG resulted in total drying of the liquid material on the sample. Wherever a particularly large droplet had been, a lump of crystalline material was found. XRF measurement of the deposited germanium gave recoveries that ranged from 150 to $300 \%$ of the expected values (samples IPS-102-IV-12 and -13 in Table 4). In view of the problems with sample drying that were encountered in our early work with synthetic samples, and based on the results of the microscopic examination of the samples, we first suspected that these high recoveries might be due to incomplete water removal by the silica gel desiccant. We reasoned that perhaps the desiccant had been used too long and was ineffective. To test this possibility, a second desiccator charged with a fresh batch of silica gel was prepared. Synthetic samples from an aqueous solution of $\mathrm{NH}_{4} \mathrm{HSO}_{4}$ were dried in both the old and new desiccators and neutralized with TAPG. In both cases, the salt appeared dry when removed from the desiccator. The acid recoveries (determined by XRF) from the old and new desiccators were $107 \%$ and $104 \%$, respectively, and demonstrated that the desiccant was not the source of the problem. 
The fairly reproducible recoveries within each set of samples lead us to believe that the problems encountered are a reflection of some real property of the samples rather than merely an erratic malfunction in the neutralization process. The occurrence of high results in these samples is best classified as an interference effect. At the present time, the sources of this interference have not been identified, but some possibilities may be suggested:

(1) The interference may be due to reaction with ammonium ion. It is conceivable that, although silica gel is an adequate desiccant for pure sulfates, a mixture of $\mathrm{H}_{2} \mathrm{SO}_{4}$ and $\mathrm{NH}_{4} \mathrm{HSO}_{4}$ might retain water more efficiently than either pure compound. This water could allow reaction with $\mathrm{NH}_{4}$ as described earlier. Alternatively, $\mathrm{H}_{2} \mathrm{SO}_{4}$ may serve as an efficient proton transfer agent if it is present in the samples in an unneutralized state. Some light might be shed on these possibilities by characterizing the liquid found in abundance in the deviant samples.

(2) The interference may be due to weak acids present in the samples. Weak acids would not dissociate in the acidic leach solution used for the reference method, but may react with the relatively strong TAPG base. Possible candidates are bisulfite ion, HS ${ }^{-}$, or amphoteric metal ions such as $\mathrm{Al}^{3+}$ or $\mathrm{Fe}^{3+}$. We have looked at the samples by $\mathrm{X}$-ray fluorescence and have not found any indication of heavy metals. FTIR analysis of these and similar samples have not indicated the presence of bisulfite, HS ${ }^{-}$, or of organic acids. However, these examinations have not ruled out the presence of weak acids in these samples.

(3) The interference may be due to complex chemical reactions between the TAPG reagent and sample constituents; such reactions could be catalyzed by the seemingly inert components of the aerosol particulates such as soot.

\section{b. Filter Samples}

The TAPG neutralization method for sulfate acidity was also investigated using laboratory-generated aerosol samples deposited on F1uoropore filters. In these experiments, a Kevex Mode1 7000 energy-dispersive $X$-ray spectrometer and data system was used for the XRF measurements. Germanium on the filters was determined using the $\mathrm{Ge}-\mathrm{K}_{\alpha}$ line at $9.88 \mathrm{KeV}$; excitation was from the $\mathrm{Br}-\mathrm{K}_{\alpha}$ line at $11.9 \mathrm{KeV}$ of a $\mathrm{KBr}$ secondary target. In addition, a titanium secondary target was employed for determining sulfur using the $S-K_{\alpha}$ line at $2.31 \mathrm{KeV}$. The ability to determine sulfur with the Kevex XRF instrument provides a useful internal standard for the germanium measurements associated with the TAPG method. Comparison of the germanium deposited on the filter during neutralization against the sulfur present on the filter provides a simple indicator of the apparent relative acidity of the sulfate aerosol.

The XRF sulfur measurement was calibrated using almost neutral sulfate aerosol deposited on 90-mm-dia Fluoropore filters. The aerosol samples were generated by thermal decomposition of ammonium sulfate in an acidic sulfate aerosol generator. ${ }^{9}$ The filters were stored over silica gel and weighed prior to depositing the aerosol. After a sample was collected, the 
filter was dried over silica gel and reweighed. From the mass of material collected and the area of the filter, the concentration of sulfate (in $\mu \mathrm{mol} / \mathrm{cm}^{2}$ ) was calculated. Figure 10 shows a plot of the sulfur counts accumulated in 200 seconds vs. the concentrations of sulfate on the filters. The straight line in this plot is the calibration line used for sulfate measurement in subsequent experiments with unknown samples.

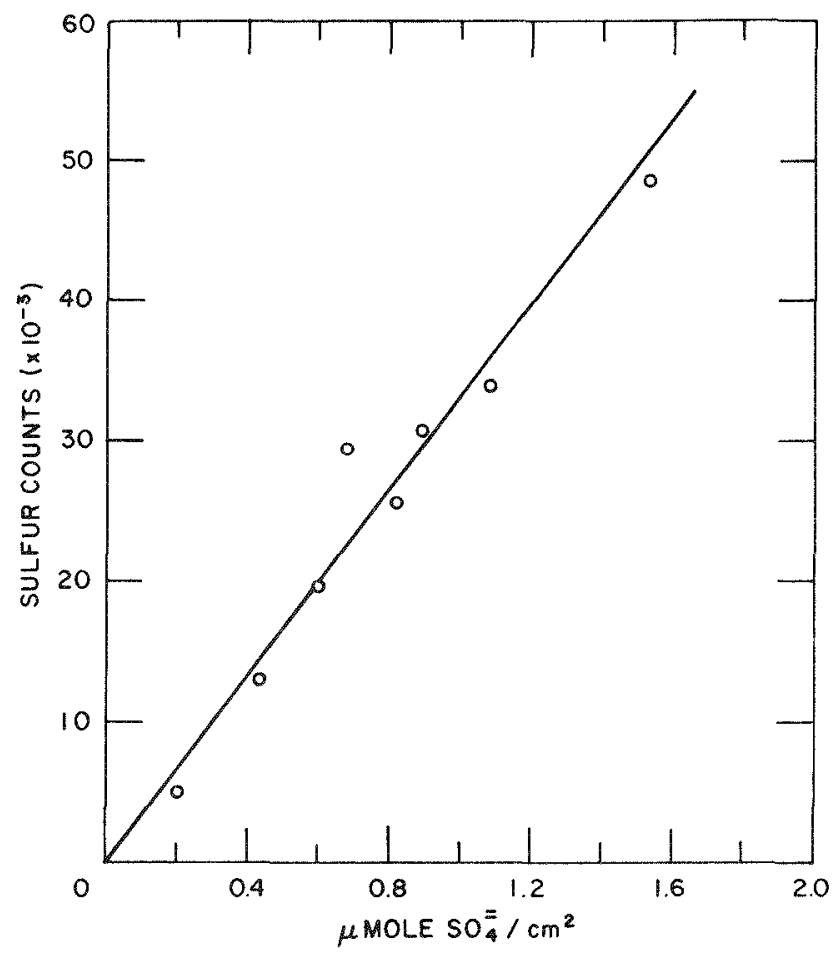

Fig. 10. Calibration of Filter Samples for Sulfur Determination by the XRF Method

The TAPG neutralization and germanium determination were evaluated using sulfuric acid aerosol samples produced by nebulizing $0.05 \mathrm{~N}$ and $0.1 \mathrm{~N} \mathrm{H}_{2} \mathrm{SO}_{4}$ solutions. Samples from the nebulizer were collected at $15 \mathrm{~L} / \mathrm{min}$ on $90-\mathrm{mm}$-dia filters. These filters were dried over silica gel and divided; portions were neutralized with TAPG and subjected to XRF analysis. As illustrated in Fig. 11, good correlation between the XRF germanium measurements and the corresponding sulfur results were obtained. This correlation is indicative of reproducible recovery of the acid in $\mathrm{H}_{2} \mathrm{SO}_{4}$ aerosol by the TAPG neutralization when the aerosol is collected on filters. However, the major difficulty encountered in applying the method to impactor samples was not that of achieving complete neutralization of sulfuric acid, but of controlling unwanted reaction with weak acids--particularly ammonium ion. 


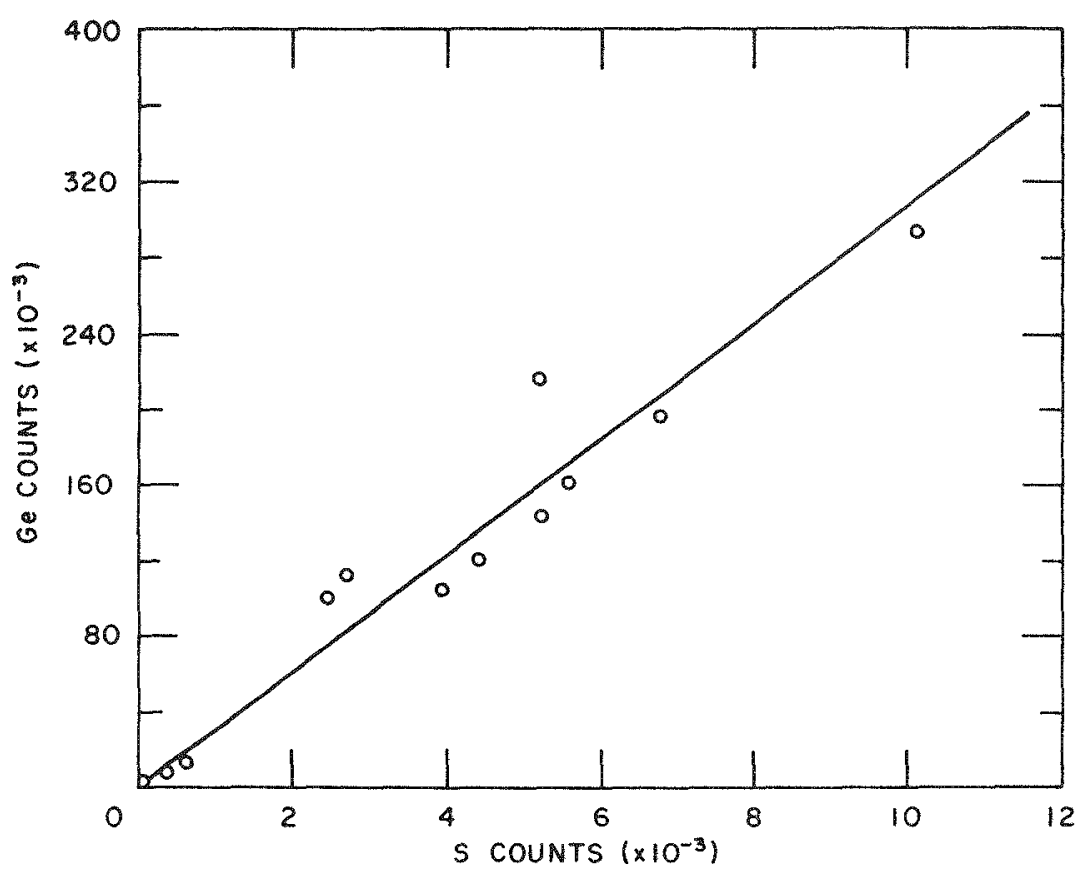

Fig. 11. Results of the Neutralization of Sulfuric Acid by TAPG on Fluoropore Filters

In order to test the possibility of ammonium ion interference when filter samples are neutralized with TAPG, a series of filters of varying acidity were prepared using the acidic sulfate aerosol generator. These filters were neutralized in the flanged-glass chamber previously employed with the impactor-type samples. The results of this experiment are shown in Table 5. The data presented there include the amount of sulfate on each filter as determined by XRF, the acidity of the sulfate measured by the $\mathrm{pH}$ change of a leach solution, the ammonium ion obtained by difference, the germanium found by XRF after TAPG neutralization, and the percent $\mathrm{NH}_{4}^{+}$that must have reacted to account for the difference between the acid on the filter and the germanium recovered. On the average, $64 \%$ of the $\mathrm{NH}_{4}^{+}$present reacted with the TAPG reagent. Clearly, under the neutralization conditions used in this experiment, interference by ammonium ion in the filters is excessive. Apparently, the material collected on filters is much more reactive than that deposited by drying of solutions or by impaction, and the conditions established using samples prepared by these latter methods are too severe for neutralization of the filter samples.

Consequently, an alternative neutralization procedure was tested in which the TAPG reagent was injected into a stream of clean, dry air flowing through the filter sample. The injection port, a glass tee fitted with a rubber serum cap, was heated to approximately $100^{\circ} \mathrm{C}$ to speed up vaporization of the amine reagent. Following injection of the amine, the air flow was continued for a few minutes to ensure removal of excess TAPG from the filter. 
Table 5. Results of Analysis of Synthetic Sulfate Aerosol on Filters Neutralized with TAPG in a Closed Chamber

\begin{tabular}{cccccc}
\hline $\begin{array}{c}\text { Sample } \\
\text { No. }\end{array}$ & $\begin{array}{c}\mathrm{H}^{+}, \mathrm{a} \\
\mu \mathrm{eq} / \mathrm{cm}^{2}\end{array}$ & $\begin{array}{c}\mathrm{SO}_{4}^{2-\mathrm{b}} \\
\mu \mathrm{mo1} / \mathrm{cm}^{2}\end{array}$ & $\begin{array}{c}\mathrm{NH}_{4}^{+}, \mathrm{c}^{2} \\
\mu \mathrm{eq} / \mathrm{cm}^{2}\end{array}$ & $\begin{array}{c}\mathrm{Ge}, \\
\mu \mathrm{eq} / \mathrm{cm}^{2}\end{array}$ & $\begin{array}{c}\mathrm{NH}_{4}^{+} \\
\text {WSJ-1 }\end{array} \mathbf{R e a c t e d}_{\%}$ \\
-2 & 0.277 & 0.566 & 0.835 & 0.928 & 78 \\
-6 & 0.235 & 0.447 & 0.659 & 0.741 & 63 \\
-8 & 0.019 & 0.030 & 0.041 & 0.049 & 77 \\
-9 & 0.001 & 0.006 & 0.011 & 0.009 & 73 \\
-10 & 0.004 & 0.006 & 0.008 & 0.009 & 73 \\
-11 & 0.0 & 0.003 & 0.006 & 0.002 & 63 \\
-12 & 0.002 & 0.008 & 0.014 & 0.009 & 33 \\
-13 & 0.032 & 0.096 & 0.160 & 0.137 & 50 \\
-14 & 0.0 & 0.010 & 0.020 & 0.010 & 66 \\
-15 & 0.188 & 0.503 & 0.818 & 0.910 & 50 \\
-16 & 0.011 & 0.187 & 0.363 & 0.213 & 88 \\
\hline
\end{tabular}

$a_{B y} \mathrm{pH}$ change of leach solution after sample addition.

${ }^{b}$ By sulfur XRF.

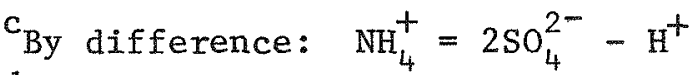

$\mathrm{d}_{\text {By }} \mathrm{Ge}$ XRF after TAPG exposure.

The results obtained with a series of weakly acidic samples using the flow-through procedure are presented in Table 6. For these samples, reaction with ammonium ion was much less pronounced than for samples neutralized in the closed chamber. Nevertheless, the interference resulting from an average $12 \%$ reaction of the $\mathrm{NH}_{4}^{+}$on the filters was sufficient to obscure any relationship between the germanium recovered and the acidity of the samples.

No other modifications of the neutralization procedure have been investigated. It is possible that optimization of the conditions for exposing the filters to TAPG might reduce the interference to acceptable levels. The probability of achieving a viable procedure appears to be higher for filter samples than for impactor samples.

\section{Summary}

A new procedure for determining the proton acidity of atmospheric aerosol samples has been examined. In this method the collected sample is exposed to a vapor-phase, tagged proton acceptor, and the amount of the tag element in the neutralized product is then measured by $X$-ray fluorescence. 
Table 6. Results of Analysis of Synthetic Sulfate Aerosol on Filters Neutralized with TAPG in Flow-Through Procedure

\begin{tabular}{|c|c|c|c|c|c|}
\hline $\begin{array}{c}\text { Sample } \\
\text { No. }\end{array}$ & $\begin{array}{c}\mathrm{H}^{+},{ }^{\mathrm{a}} \\
\mu \mathrm{eq} / \mathrm{cm}^{2}\end{array}$ & $\begin{array}{c}\mathrm{SO}_{4}^{2-}, \mathrm{b} \\
\mu \mathrm{mol} / \mathrm{cm}^{2}\end{array}$ & $\begin{array}{c}\mathrm{NH}_{4}^{+}, \mathrm{c} \\
\mu \mathrm{eq} / \mathrm{cm}^{2}\end{array}$ & $\begin{array}{c}\mathrm{Ge},{ }^{\mathrm{d}} \\
\mu \mathrm{eq} / \mathrm{cm}^{2}\end{array}$ & $\underset{\%}{\mathrm{NH}^{+}}$Reacted, \\
\hline WSJ-31 & 0.150 & 0.924 & 1.697 & 0.258 & 6 \\
\hline-32 & 0.085 & 0.884 & 1.683 & 0.108 & 1 \\
\hline-33 & 0.009 & 0.767 & 1.525 & 0.111 & 7 \\
\hline-34 & 0.0 & 0.151 & 0.302 & 0.070 & 23 \\
\hline-40 & 0.248 & 1.463 & 2.679 & 0.323 & 3 \\
\hline-41 & 0.213 & 0.583 & 0.953 & 0.320 & 11 \\
\hline-42 & 0.027 & 1.018 & 2.010 & 0.314 & 14 \\
\hline-44 & 0.006 & 0.398 & 0.789 & 0.275 & 34 \\
\hline
\end{tabular}

${ }^{a} \mathrm{By}$ pH change of leach solution after sample addition.

${ }_{B}$ By sulfur XRF.

${ }^{c}$ By difference.

$\mathrm{d}_{\text {By }}$ Ge XRF after TAPG exposure.

On the basis of desirable physical and chemical properties, trimethyl-3aminopropy1-germane was selected as the proton acceptor. Tests were conducted with both impactor-collected and filter-collected samples. It was found that both the response of the method to proton acidity and the extent of interference from the $\mathrm{NH}_{4}^{+}$ion were strongly affected by sample preparation and neutralization conditions. These conditions could be optimized for synthetic samples containing $\mathrm{H}_{2} \mathrm{SO}_{4}, \mathrm{NH}_{4} \mathrm{HSO}_{4}$, and $\left(\mathrm{NH}_{4}\right)_{2} \mathrm{SO}_{4}$. However, these optimized conditions were still not suitable for samples collected in the field, for which the procedure gave almost quantitative response for the $\mathrm{NH}_{4}^{+}$ ion, as well as for the $\mathrm{H}^{+}$ions present. The detailed nature of this interference has not yet been determined.

\section{ACKNOWLEDGMENTS}

The authors are grateful to Jeremey (Jake) Hales of Battelle Pacific Northwest Laboratory for use of their DC-3 aircraft in the airborne tests of the ATR-impactor. We also gratefully acknowledge the supporting efforts of W. S. Jones and $E$. T. Kucera in sample preparation and analysis for the $\mathrm{X}$-ray fluorescence method for proton acidity. The work described in this report was funded primarily by the U.S. Environmental Protection Agency, and partly by the U.S. Department of Energy, Division of Basic Energy Sciences Research. 
REFERENCES

1. P. T. Cunningham, S. A. Johnson, and R. T. Yang, Environ. Sci. Technol. 8, 131 (1974).

2. P. T. Cunningham and S. A. Johnson, Science 191, 77-79 (January 1976).

3. I. Citron and A. L. Underwood, Anal. Chem. Acta 22, 338-344 (1960).

4. R. J. Char1son, A. H. Vanderpool, D. S. Covert, A. P. Waggoner, and N. C. Ahlquist, Science 184, 156-157 (April 1974).

5. W. G. Cobourn, R. B. Husar, and J. D. Husar, Atmos, Environ. 12, 89-98 (1978).

6. N. J. Harrick, Internal Reflection Spectroscopy, Interscience Publishers, New York, 1967.

7. V. A. Marple and $\mathrm{K}$. Willeke, Inertial Impactors: Theory, Design and Use, Fine Particle Symposium, Minneapolis, Minnesota, May 28-30, 1975, Particle Technology Laboratory Publication No. 251 ,

8. C. Huygens, Atmos. Environ. 9, 315-319 (1975).

9. G. M. Kanapilly and K. W. Tu, Inhalation Toxicology Research Institute Annual Report for 1976-1977, Lovelace Biomedical and Environmental Research Institute, pp. 273-279 (December 1977). 
Distribution for ANL-81-12

Interna1:
L. Burris
D. W. Green
D. M. Rote
E. J. Croke
C. C. Marcelo
D. L. Sisterson
J. J. Roberts
E. T. Kucera
J. D. Shannon
R. E. Rowland
S. Siege1
W. K. Sinclair
B. D. Holt
M. L. Wesely
F. A. Cafasso
D. L. Drapcho
C. M. Sheih
P. T. Cunningham
R. J. Meyer
S. A. Johnson (10)
K. J. Jensen
R. Kumar
R. R. Heinrich
D. G. Graczyk
P. R. Raptis
R. P. Larsen
P. Frenzen
A. F. Melton
V. A. Maroni
J. E. Harmon
ANL Patent Dept.
ANL Contract File
ANL Libraries (2)
D. S. Webster
TIS Files (6)

\section{Externa :}

DOE-TIC, for distribution per UC-11 (233)

Manager, Chicago Operations Office, DOE

Director, Technology Management Div., DOE-CH

Argonne Universities Association:

President

C. B. Alcock, U. Toronto

R. C. Axtmann, Princeton U.

P. W. Gilles, U. Kansas

R. I. Newman, Fripp Island, S. C.

R. Charlson, U. Washington

R. DePena, Pennsylvania State U.

J. Durham, U. S. Environmental Protection Agency, Research Triangle Park

T. Dzubay, U. S. Environmental Protection Agency, Research Triangle Park

D. Eatough, Brigham Young U.

J. Galloway, U. Virginia

J. Hales, Pacific Northwest Lab.

N. J. Harrick, Ossining, N. Y.

B. Hicks, National Oceanic and Atmospheric Adm., Oak Ridge

R. B. Husar, Washington U.

W. S. Jones, Chesapeake, Va.

L. Newman, Brookhaven National Lab.

T. Novakov, Lawrence Berkeley Lab.

C. Steen, Pittsburgh 